\title{
Genetic architecture of quantitative traits in beef cattle revealed by genome wide association studies of imputed whole genome sequence variants: Il: carcass merit traits
}

Yining Wang ${ }^{1,2}$, Feng Zhang ${ }^{1,2,3,4}$, Robert Mukiibi², Liuhong Chen ${ }^{1,2}$, Michael Vinsky ${ }^{1}$, Graham Plastow ${ }^{2}$, John Basarab ${ }^{5}$, Paul Stothard ${ }^{2}$ and Changxi Li, ${ }^{1,2^{*}}$

\begin{abstract}
Background: Genome wide association studies (GWAS) were conducted on 7,853,211 imputed whole genome sequence variants in a population of 3354 to 3984 animals from multiple beef cattle breeds for five carcass merit traits including hot carcass weight (HCW), average backfat thickness (AFAT), rib eye area (REA), lean meat yield (LMY) and carcass marbling score (CMAR). Based on the GWAS results, genetic architectures of the carcass merit traits in beef cattle were elucidated.

Results: The distributions of DNA variant allele substitution effects approximated a bell-shaped distribution for all the traits while the distribution of additive genetic variances explained by single DNA variants conformed to a scaled inverse chi-squared distribution to a greater extent. At a threshold of $P$-value $<10^{-5}, 51,33,46,40$, and 38 lead DNA variants on multiple chromosomes were significantly associated with HCW, AFAT, REA, LMY, and CMAR, respectively. In addition, lead DNA variants with potentially large pleiotropic effects on HCW, AFAT, REA, and LMY were found on chromosome 6. On average, missense variants, $3^{\prime} U T R$ variants, 5'UTR variants, and other regulatory region variants exhibited larger allele substitution effects on the traits in comparison to other functional classes. The amounts of additive genetic variance explained per DNA variant were smaller for intergenic and intron variants on all the traits whereas synonymous variants, missense variants, 3'UTR variants, 5'UTR variants, downstream and upstream gene variants, and other regulatory region variants captured a greater amount of additive genetic variance per sequence variant for one or more carcass merit traits investigated. In total, 26 enriched cellular and molecular functions were identified with lipid metabolisms, small molecular biochemistry, and carbohydrate metabolism being the most significant for the carcass merit traits.

(Continued on next page)
\end{abstract}

\footnotetext{
* Correspondence: changxi.li@canada.ca

${ }^{1}$ Lacombe Research and Development Centre, Agriculture and Agri-Food

Canada, Lacombe, AB, Canada

${ }^{2}$ Department of Agricultural, Food and Nutritional Science, University of

Alberta, Edmonton, AB, Canada

Full list of author information is available at the end of the article
} 
(Continued from previous page)

Conclusions: The GWAS results have shown that the carcass merit traits are controlled by a few DNA variants with large effects and many DNA variants with small effects. Nucleotide polymorphisms in regulatory, synonymous, and missense functional classes have relatively larger impacts per sequence variant on the variation of carcass merit traits. The genetic architecture as revealed by the GWAS will improve our understanding on genetic controls of carcass merit traits in beef cattle.

Keywords: Genetic architecture, Imputed whole genome sequence variants, Genome wide association studies, Carcass merit traits, Beef cattle,

\section{Background}

Carcass merit traits are important to beef production as they directly determine carcass yield, grade, and consumer preferences for meat consumption, and therefore profitability. Genetic improvement of carcass merit traits has been made possible by recording pedigree and/or performance data to predict genetic merit of breeding candidates. However, carcass merit traits are expressed at later stages of animal production and are mostly assessed at slaughter, which sacrifices potential breeding stock although real-time ultrasound imaging technologies can be used to measure some carcass traits such as backfat thickness, longissimus dorsi muscle area, and marbling score on live animals [1]. With the discovery of DNA variants and development of a $50 \mathrm{~K}$ SNP panel that covers the whole genome for cattle [2], utilization of DNA markers in predicting genetic merit such as genomic selection holds great promise to accelerate the rate of genetic improvement by shortening the generation interval and/or by increasing the accuracy of genetic evaluation $[3,4]$. However, the accuracy of genomic prediction for carcass traits in beef cattle still needs to be improved for wider industry application of genomic selection [5-7]. Although collection of more data on relevant animals to increase the reference population size will improve the genomic prediction accuracy, better understanding on genetic architecture underlying complex traits such as carcass merit traits will help develop a more effective genomic prediction strategy to further enhance feasibility of genomic selection in beef cattle $[8,9]$.

Early attempts to understanding the genetic control of quantitative traits in beef cattle were made with the detection of chromosomal regions or quantitative trait loci (QTL) $[10,11]$. However, these QTLs are usually localized at relatively large chromosomal regions due to relatively low density DNA marker panels used at the time $[8,12,13]$. With the availability of the bovine $50 \mathrm{~K} \mathrm{SNP}$ chips [2] and high density (HD) SNPs (Axiom ${ }^{\text {тs }}$ Genome-Wide BOS 1 Bovine Array from Affymetrix $₫$, USA, termed "HD" or "AffyHD" hereafter), identification of significant SNPs associated with carcass merit traits have led to better fine-mapped QTL regions. All these studies have resulted in multiple QTL candidates for carcass traits in beef cattle, and an extensive QTL database has been created and is available at the Cattle QTL database [14]. In addition, identification of causative mutations underlying the QTL regions has been attempted through association analyses between selected positional and functional candidate gene markers and the traits [10, 15-21]. These identified QTLs and candidate gene markers have improved our understanding on the genetic influence of DNA variants on carcass traits in beef cattle. However, the genetic architecture including causal DNA variants that control the carcass traits still remains largely unknown.

The recent discovery and functional annotation of tens of millions of DNA variants in cattle has offered new opportunities to investigate whole genome wide sequence variants associated with complex traits in beef cattle [22]. The whole genome sequence (WGS) variants represent the ideal DNA marker panel for genetic analyses as they theoretically contain all causative polymorphisms. Although whole genome sequencing on a large number of samples may be impractical and cost prohibitive at present, imputation of SNPs from genotyped lowerdensity DNA panels such as the $50 \mathrm{~K}$ SNP panel up to the WGS level may provide a valuable DNA marker panel for genetic analyses including GWAS due to its high DNA marker density. In a companion study, we imputed the bovine $50 \mathrm{~K}$ SNP genotypes to whole genome sequence (WGS) variants for 11,448 beef cattle of multiple Canadian beef cattle populations and retained 7,853,211 DNA variants for genetic/genomic analyses after data quality control of the imputed WGS variants [23]. We also reported the GWAS results for feed efficiency and its component traits based on the 7,853,211 DNA variants in a multibreed population of Canadian beef cattle [23]. The objective of this study was to further investigate the effects of the imputed 7,853,211 WGS DNA variants (or termed as 7.8 M DNA variants or $7.8 \mathrm{M}$ SNPs in the text for simplicity) on carcass merit traits including hot carcass weight ( $\mathrm{HCW})$, average backfat thickness (AFAT), rib eye area (REA), lean meat yield (LMY), and carcass marbling score (CMAR). 


\section{Results}

Descriptive statistics and heritability estimates for carcass merit traits

Means and standard deviations of raw phenotypic values for the five carcass merit traits in this study (Table 1) are in line with those previously reported in Canadian beef cattle populations [24, 25]. Heritability estimates of the five carcass merit traits based on the marker-based genomic relationship matrix (GRM) constructed with the $50 \mathrm{~K}$ SNP panel ranged from $0.28 \pm 0.03$ for AFAT to $0.40 \pm 0.03$ for $\mathrm{HCW}$ (Table 1). With the GRMs of the imputed 7.8 M DNA variants, we observed increased heritability estimates for all the five investigated traits, ranging from $0.33 \pm 0.03$ to $0.35 \pm 0.04$ (or 6.1\% increase) for LMY and from $0.40 \pm 0.03$ to $0.49 \pm 0.03$ (or $22.5 \%$ increase) for HCW without considering their SE. These corresponded to an increase in additive genetic variances explained by the $7.8 \mathrm{M}$ DNA variants from $5.7 \%$ for LMY to $24.0 \%$ for HCW, which indicated that the imputed 7.8 M DNA variants were able to capture more genetic variance than the $50 \mathrm{~K}$ SNP panel, with different scales of increment depending on the trait. DNA marker-based heritability estimates for all five traits using both $50 \mathrm{~K}$ SNPs and imputed 7.8 M DNA variants are slightly smaller than the pedigree based heritability estimates that were obtained from a subset of animals from the population [24], suggesting that neither the 50 $\mathrm{K}$ SNP panel nor the $7.8 \mathrm{M}$ DNA variants may capture the full additive genetic variance.

\section{Comparison of GWAS results between $7.8 \mathrm{M}$ and $50 \mathrm{~K}$ SNP panels}

At the suggestive threshold of $P$-value $<0.005$ as proposed by Benjamin et al. [26], the GWAS of the imputed 7.8 M SNPs detected a large number of SNPs in association with the traits, ranging from 42,446 SNPs for LMY to 45,303 SNPs for AFAT (Table 2). The numbers of additional or novel significant SNPs detected by the 7.8 M DNA panel in comparison to the $50 \mathrm{~K}$ SNP GWAS were presented in Table 2, ranging from 31,909 for REA to 34,227 for AFAT. The majority of the suggestive SNPs identified by the $50 \mathrm{~K}$ SNP panel GWAS for the five carcass merit traits (ranging from $85 \%$ for AFAT to 91\% for CMAR) were also detected by the imputed 7.8 M SNP GWAS at the threshold of $P$-value $<0.005$. Further investigation showed that all of these suggestive significant SNPs detected by the $50 \mathrm{~K}$ SNP panel GWAS were also significant by the 7.8 M SNP GWAS if the significance threshold was relaxed to $P$-value $<0.01$, indicating that the imputed $7.8 \mathrm{M}$ SNP panel GWAS was able to detect all the significant SNPs of the $50 \mathrm{~K} \mathrm{SNP}$ panel. The small discrepancy in P-values of each SNP between the two DNA variant panels is likely due to the different genomic relationship matrices used. This result is expected as the 7.8 M DNA variant panel included all SNPs in the $50 \mathrm{~K}$ panel and this study used a single marker based model for GWAS. These additional or novel significant SNPs detected by the $7.8 \mathrm{M}$ DNA marker panel corresponded to the increased amount of additive genetic variance captured by the $7.8 \mathrm{M}$ DNA variants in comparison to the $50 \mathrm{~K}$ SNP panel, indicating that the imputed $7.8 \mathrm{M}$ DNA variants improved the power of GWAS for the traits. Therefore, we will focus on the GWAS results of the $7.8 \mathrm{M}$ DNA variants in subsequent result sections.

\section{DNA marker effects and additive genetic variance related to functional classes}

Plots of the allele substitution effects of imputed 7,853, 211 WGS variants showed a bell-shaped distribution for all the traits (Additional file 1: Figure S1). Distributions of additive genetic variances explained by single DNA variants followed a scaled inverse chi-squared distribution for all the five traits to a greater extent (Additional file 1: Figure S1). When the DNA marker or SNP effects of the 9 functional classes were examined, differences in their average squared SNP allele substitution effects were observed as shown in Table 3 . In general, missense variants, 3'UTR, 5'UTR, and other regulatory region variants exhibited a larger effect on all five carcass merit traits investigated in comparison to DNA variants in other functional classes. Intergenic variants and intron variants captured a greater amount of total additive genetic variance for all five carcass traits. However, the

Table 1 Descriptive statistics of phenotypic data, additive genetic variances and heritability estimates based on the $50 \mathrm{~K}$ SNP and the imputed $7.8 \mathrm{M}$ whole genome sequence (WGS) variants in a beef cattle multibreed population for carcass merit traits

\begin{tabular}{lllllll}
\hline Traits $^{\mathrm{a}}$ & $\mathrm{n}$ & mean (sd) & $50 \mathrm{~K} \sigma_{a}^{2} \pm S E$ & $50 \mathrm{~K} h^{2} \pm$ SE & $7.8 \mathrm{M} \sigma_{a}^{2} \pm S E$ & $7.8 \mathrm{M} \mathrm{h} \pm$ SE \\
\hline HCW & 3984 & $337.26(35.42)$ & $335.77 \pm 23.39$ & $0.40 \pm 0.03$ & $416.26 \pm 35.60$ & $0.49 \pm 0.03$ \\
AFAT & 3354 & $11.11(4.70)$ & $3.15 \pm 0.35$ & $0.28 \pm 0.03$ & $3.52 \pm 0.50$ & $0.32 \pm 0.04$ \\
REA & 3979 & $85.46(11.92)$ & $28.15 \pm 2.19$ & $0.36 \pm 0.03$ & $32.96 \pm 3.34$ & $0.42 \pm 0.03$ \\
LMY & 3367 & $57.43(5.02)$ & $3.49 \pm 0.34$ & $0.33 \pm 0.03$ & $3.69 \pm 0.49$ & $0.35 \pm 0.04$ \\
CMAR & 3928 & $406(89)$ & $1136.98 \pm 104.48$ & $0.29 \pm 0.03$ & $1326.30 \pm 156.30$ & $0.34 \pm 0.03$ \\
\hline
\end{tabular}

${ }^{a} H C W$ hot carcass weight in $\mathrm{kg}, A F A T$ average backfat thickness in $\mathrm{mm}, R E A$ rib eye area in $\mathrm{cm}^{2}$, LMY lean meat yield in \%, CMAR carcass marbling score from 100 (trace marbling) to 499 (more marbling). mean (SD) = mean of raw phenotypic values and standard deviation $(\mathrm{SD}), \sigma_{\mathrm{a}}{ }^{2} \pm \mathrm{SE}=\mathrm{additive}$ genetic variance \pm standard error (SE), $\mathrm{h}^{2} \pm \mathrm{SE}=$ heritability estimate $\pm \mathrm{SE}$ 
Table 2 A summary of number of significant DNA variants detected by the 7.8 M WGS variant GWAS for carcass merit traits in a beef cattle multibreed population

\begin{tabular}{llllll}
\hline Trait $^{\text {a }}$ & HCW & AFAT & REA & LMY & CMAR \\
\hline Suggestive $(p<0.005)$ & $42,612(32,240)$ & $45,303(34,227)$ & $42,544(31,909)$ & $42,446(33,305)$ & $44,654(33,211)$ \\
Lead Suggestive & $3927(3621)$ & $3922(3598)$ & $3993(3705)$ & $3906(3606)$ & $4158(3827)$ \\
Significant $\left(p<10^{-5}\right)$ & $1413(374)$ & $260(162)$ & $1171(254)$ & $312(198)$ & $256(145)$ \\
Lead Significant & $51(27)$ & $33(23)$ & $46(25)$ & $40(31)$ & $38(28)$ \\
FDR (FDR $<0.10)$ & $1997(374)$ & $183(97)$ & $1255(254)$ & $168(86)$ & $107(59)$ \\
Lead FDR (FDR $<0.10)$ & $51(27)$ & $15(9)$ & $46(25)$ & $16(11)$ & $12(8)$
\end{tabular}

${ }^{a} \mathrm{HCW}$ hot carcass weight in $\mathrm{kg}$, AFAT average backfat thickness in $\mathrm{mm}, R E A$ rib eye area in $\mathrm{cm}^{2}$, LMY lean meat yield in \%, CMAR carcass marbling score from 100 (trace marbling) to 499 (more marbling). FDR = genome-wise false discovery rate (FDR) calculated from the Benjamini-Hochberg procedure [27]. The numbers of additional or novel significant SNPs in comparison to the $50 \mathrm{~K}$ SNP panel were presented in the parentheses

relative proportion of additive genetic variance explained per sequence variant by intergenic and intron variants was smaller than those of other functional classes. Relatively, missense variants captured a greater amount of additive genetic variance per sequence variant for REA, LMY, and CMAR while 3'UTR explained more additive genetic variance per DNA variant for $\mathrm{HCW}$, AFAT, and REA. DNA variants in 5'UTR and other regulatory region variants also showed a greater amount of additive genetic variance explained per sequence variant for CMAR and for CMAR and REA, respectively. Although synonymous variants had relatively smaller averages of squared SNP allele substitution effects, a single DNA variant in the synonymous functional class accounted for more additive genetic variance for AFAT, REA, LMY and CMAR. In addition, both the downstream and upstream gene variants were found to capture more additive genetic variance per sequence variant for HCW (Table 3).

\section{Top significant SNPs associated with carcass merit traits} The suggestive lead SNPs associated with HCW, AFAT, REA, LMY, and CMAR in Table 2 were distributed across all the autosomes as shown in the Manhattan plots of 7.8 M DNA variant GWAS (Fig. 1). The numbers of lead SNPs were dropped to 51, 33, 46, 40, and 38 for HCW, AFAT, REA, LMY, and CMAR, respectively, at a more stringent threshold of $P$-value $<10^{-5}$, of which $51,15,46,16$, and 12 lead significant SNPs reached a FDR $<0.10$ for HCW, AFAT, REA, LMY, and CMAR, respectively (Table 2).

The lead significant SNPs at the nominal $P$-value $<10^{-5}$ for the five carcass merit traits were distributed on multiple autosomes (Fig. 2). In general, SNP with larger effects were observed on BTA6 for HCW, AFAT, LMY, and REA. For CMAR, SNPs with relatively larger effects were located on BTA1 and BTA2 (Additional file 2). To show lead SNPs on each chromosome, Table 4 lists top significant lead SNPs with larger phenotypic variance explained on each chromosome. The top lead variant Chr6:39111019 for HCW on BTA6 was an INDEL located 118,907 bp from gene $L C O R L$ and explained $4.79 \%$ of the phenotypic variance. SNP rs109658371 was another lead SNP on BTA6 and it explained $4.65 \%$ of phenotypic variance for HCW. Additionally, SNP rs109658371 was located 102,547 bp upstream of the top SNP Chr6:39111019 and it is 221,454 bp away from the nearest gene LCORL. Outside BTA6, two other SNPs rs109815800 and rs41934045 also had relatively large effects on HCW, explaining 3.41 and $1.47 \%$ of phenotypic variance and are located on BTA14 and BTA20, respectively. SNPs rs 109815800 is 6344 bp away from gene PLAG1 whereas SNP rs41934045 is located in the intronic region of gene ERGIC1. For AFAT, two lead SNPs explaining more than $1 \%$ of phenotypic variance included SNP rs110995268 and SNP rs41594006. SNP rs110995268 is located in the intronic region of gene $L C O R L$ on BTA6, explaining $2.87 \%$ of phenotypic variance. SNP rs41594006, which explained $1.07 \%$ of phenotypic variance, is 133,040 bp away from gene MACC1 on BTA4. SNPs $r s 109658371$ and $r s 109901274$ are the two lead SNPs on different chromosomes that explained more than $1 \%$ of phenotypic variance for REA. These two lead SNPs are located on BTA6 and BTA7, respectively. SNP rs 109658371 accounted for $3.32 \%$ of phenotypic variance for REA and is $221,454 \mathrm{bp}$ away from gene LCORL while SNP rs109901274 is a missense variant of gene ARRDC3, explaining $1.11 \%$ of phenotypic variance for REA. For LMY, SNPs rs380838173 and rs110302982 are the two lead SNPs with relatively larger effects. Both SNPs are located on BTA6, explaining 2.59 and $2.53 \%$ of phenotypic variance respectively. SNP rs 380838173 is $128,272 \mathrm{bp}$ away from gene LCORL while SNP rs110302982 is only 5080 bp away from gene NCAPG. For CMAR, two lead SNPs rs211292205 and rs441393071 on BTA1 explained 1.20 and $1.04 \%$ of phenotypic variance. SNP $r s 211292205$ is 50,986 bp away from gene MRPS6 while SNP rs441393071 was an intron SNP of gene MRPS6. The rest of the lead significant SNPs for CMAR accounted for less than $1 \%$ of phenotypic variance (Table 4).

\section{Enriched molecular and cellular and gene network}

With a window of 70kbp extending upstream and downstream of each of the lead SNPs at FDR $<0.10,319$ candidate genes for HCW, 189 for AFAT, 575 for REA, 329 


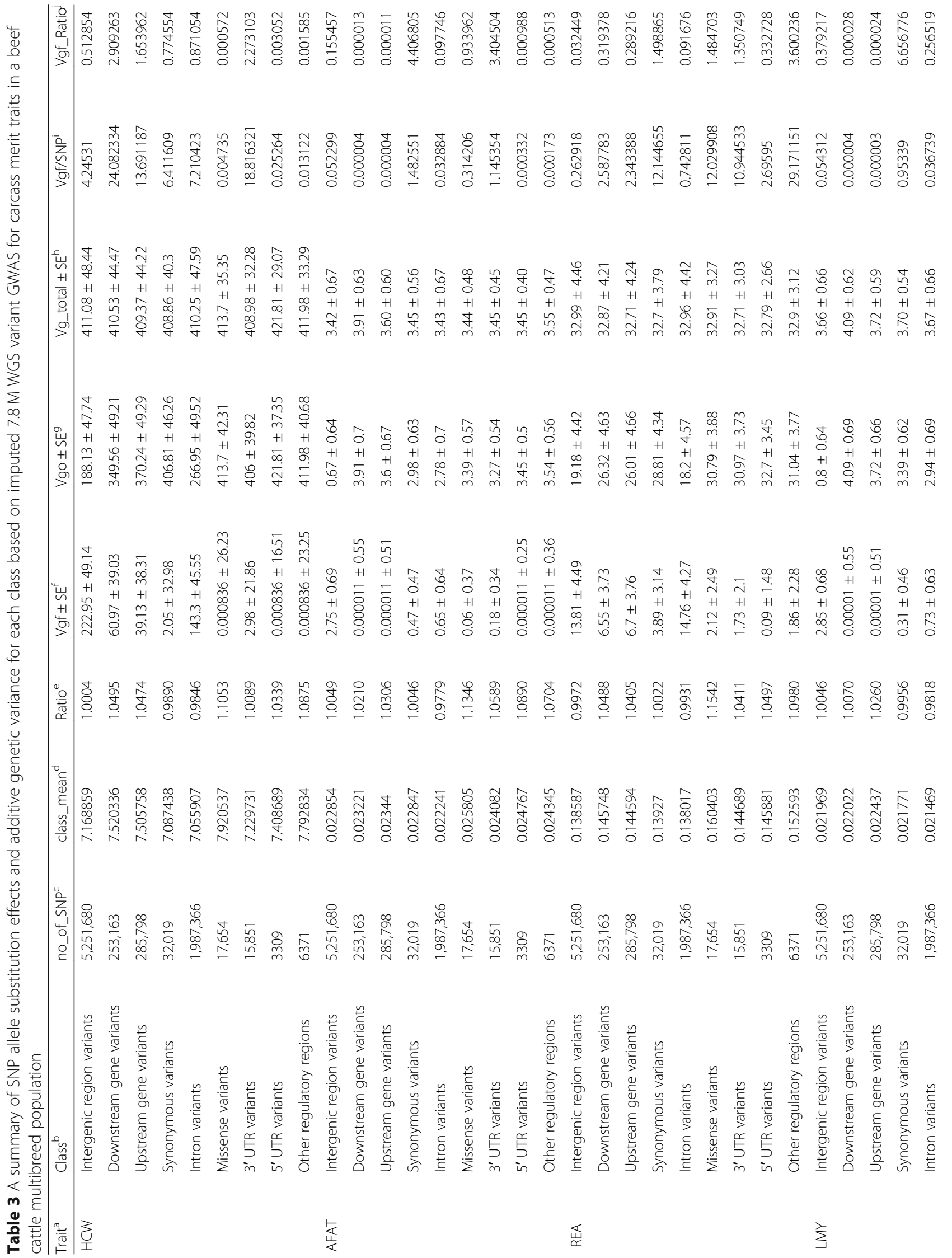




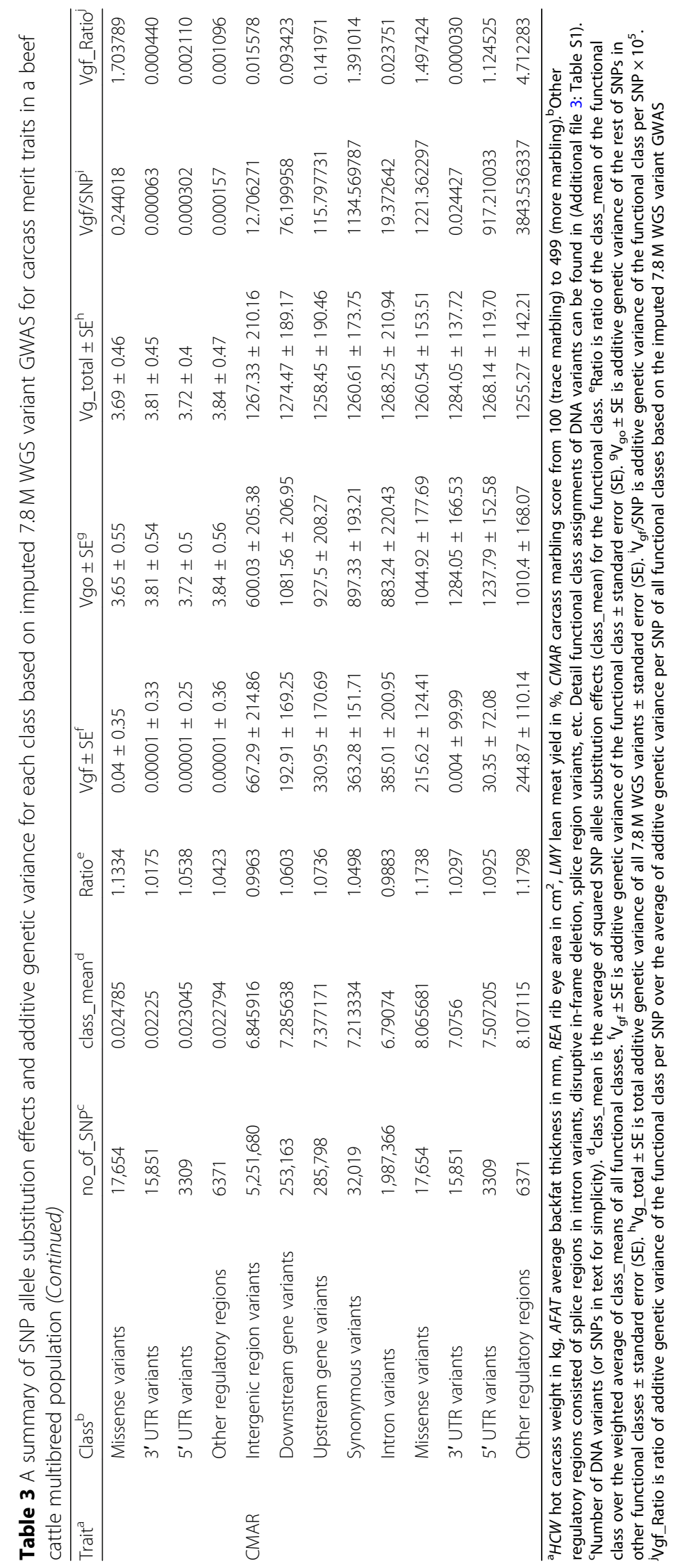




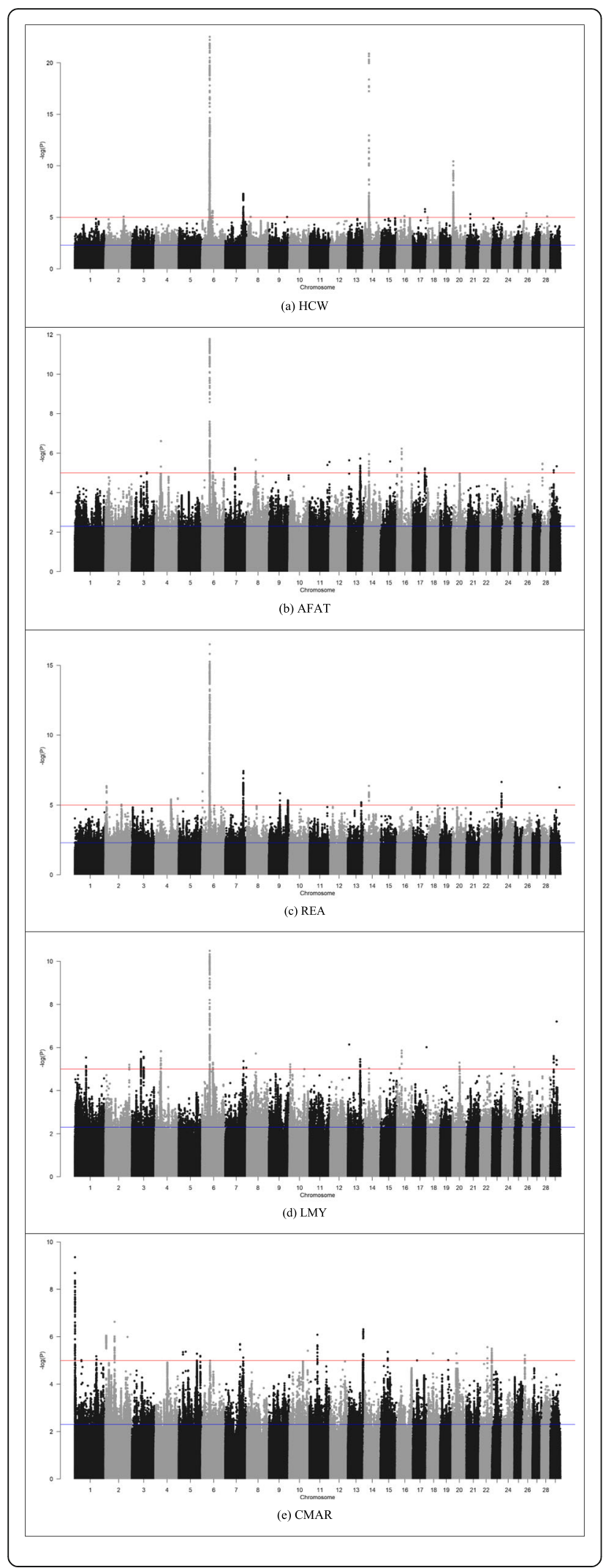

Fig. 1 Manhattan plots of GWAS results based on the imputed 7.8 M DNA variant panel for (a) hot carcass weight (HCW), (b) average backfat thickness (AFAT), (c) rib eye area (REA), (d) lean meat yield (LMY), and (e) carcass marbling score (CMAR). The vertical axis reflects the $-\log _{10}(P)$ values and the horizontal axis depicts the chromosomal positions. The blue line indicates a threshold of $P$-value $<0.005$ while the red line shows the threshold of $P$-value $<10^{-5}$

for LMY, and 198 for CMAR were identified based on annotated Bos taurus genes (23,431 genes on autosomes in total) that were downloaded from the Ensembl BioMart database (accessed on 8 November, 2018) (Additional file 1: Figure S4b). Of the identified candidate genes, 308, 180, 557, 318, and 188 genes were mapped to IPA knowledge base for HCW, AFAT, REA, LMY, and CMAR, respectively. In total, we identified 26 enriched molecular and cellular functions for AFAT, CMAR, and REA, and 25 functions for HWC and LMY at a $P$-value $<0.05$ as presented in Additional file 1: Figure S2. Of all the five traits, lipid metabolism was among the top five molecular and cellular functions for AFAT, REA, LMY, and CMAR. For HCW, lipid metabolism was the sixth highest biological function involving 46 of the candidate genes. Across the five traits, the lipid related genes are primarily involved in the synthesis of lipid, metabolism of membrane lipid derivatives, concentration of lipid, and steroid metabolism processes as shown in the gene-biological process interaction networks (Additional file 1: Figure S3). Interestingly 18 genes involved in lipid synthesis including ACSL6, CFTR, NGFR, ERLIN1, TFCP2L1, PLEKHA3, STRSIA1, PPARGC1A, MAPK1, PARD3, PLA2G2A, AGMO, MOGAT2, PIGP, PIK3CB, NR5A1, CNTFR, and BMP7 are common for all the four traits. It is also worth noting that 18 ( $A G M O$, BID, BMP7, CFTR, CLEC11A, GNAI1, MOGAT2, MRAS, NGFR, NR5A1, P2RY13, PDK2, PIK3CB, PLA2G2A, PPARGC1A, PPARGC1B, PTHLH, and ST8SIA1) of the 31 genes involved in lipid metabolism for AFAT have roles in lipid concentration.

Additionally, our results also revealed small molecular biochemistry and carbohydrate metabolism as other important molecular and cellular processes for AFAT, CMAR, HCW, and LMY (Additional file 1: Figure S3). Some of the major enriched subfunctions or biological processes related to carbohydrate metabolism included uptake of carbohydrate, synthesis of carbohydrate, and synthesis of phosphatidic acid as shown in the genebiological process interaction networks (Additional file 1: Figure S3). For REA, cell morphology, cellular assembly and organization, cellular function and maintenance are the top enriched molecular processes in addition to lipid metabolism and molecular transport. The major enriched biological processes and subfunctions related within cell morphology function included transmembrane potential, 

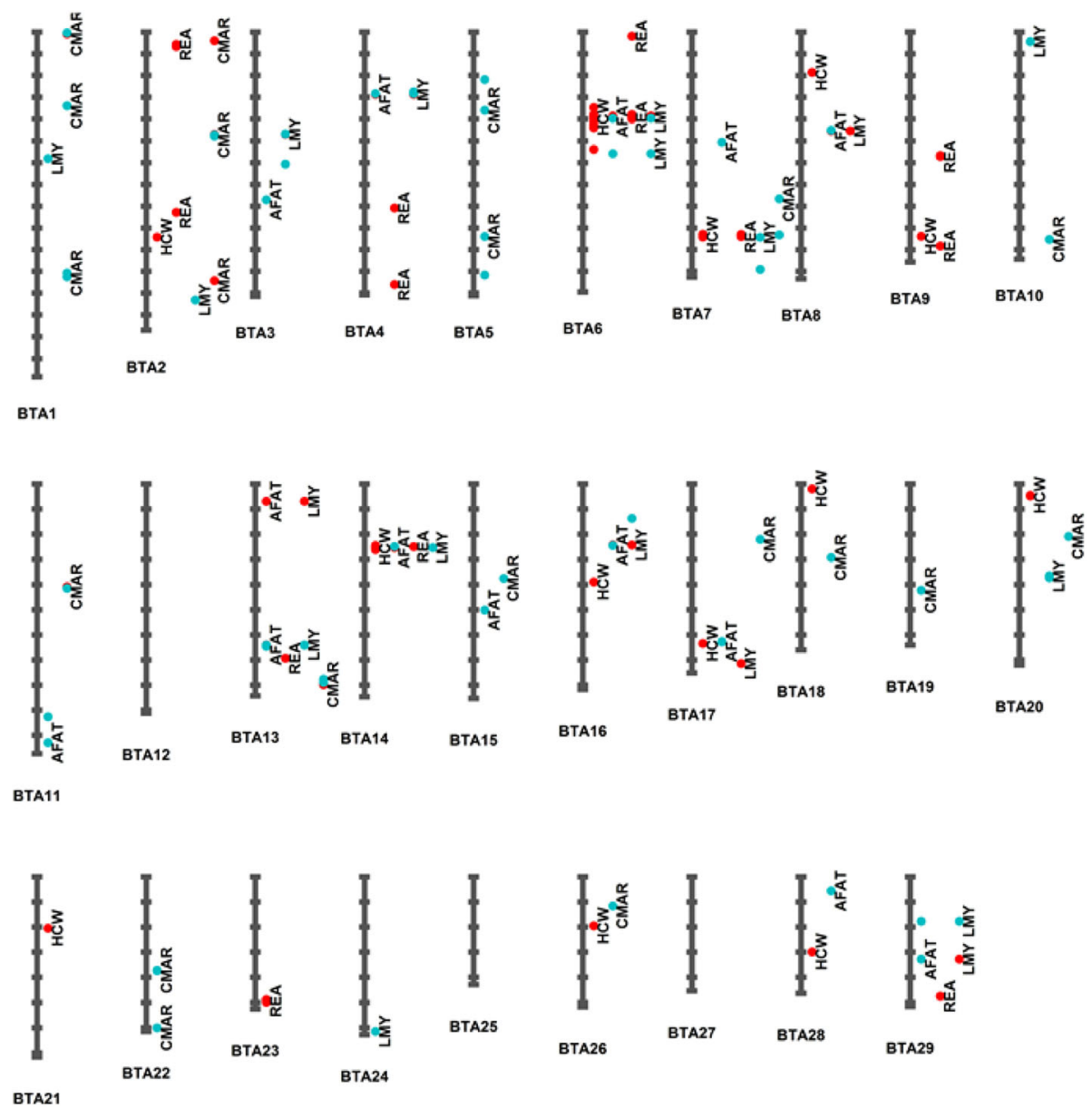

Fig. 2 Distribution of lead SNPs at P-value $<10^{-5}$ on Bos taurus autosomes (BTA) for hot carcass weight (HCW), average backfat thickness (AFAT), rib eye area (REA), lean meat yield (LMY), and carcass marbling score (CMAR). The blue dots indicate a threshold of $P$-value $<10^{-5}$ while the red dots show the threshold of both $P$-value $<10^{-5}$ and genome-wise false discovery rate (FDR) $<0.10$

transmembrane potential of mitochondria, morphology of epithelial cells, morphology of connective tissue cells, and axonogenesis as presented in (Additional file 1: Figure S3). For cellular function and maintenance, the genes are mainly involved in organization of cellular membrane, axonogenesis, the function of mitochondria, and transmembrane potential of the cellular membrane. The genes involved in these processes and subfunctions are also shown in Additional file 1: Figure S3. Table 5 lists all the genes involved in each of the top five enriched molecular processes for each trait while examples of the gene network for lipid metabolism and carbohydrate metabolism are presented in Additional file 1: Figure S3.

\section{Discussion}

The value of the imputed $7.8 \mathrm{M}$ whole genome sequence variants on GWAS

With the $50 \mathrm{~K}$ SNPs $(N=30,155)$ as the base genotypes, a reference population of 4059 animals of multi-breeds genotyped with the Affymetrix HD panel, and a panel of 1570 animals with WGS variants from run 5 of the 1000 Bull Genomes Project, we achieved an average imputation accuracy of $96.41 \%$ on $381,318,974$ whole genotype sequence variants using FImpute 2.2 [28]. This average imputation accuracy is comparable to the imputation accuracy previously obtained in beef cattle [29] but slightly lower than that in dairy cattle [30,31]. However, the imputation accuracy over a validation dataset of 240 animals varied among individual DNA variants, with a range from 0.42 to $100 \%$ (data not shown). To ensure a higher quality of imputed WGS DNA variants, we removed imputed WGS DNA variants with an average imputation accuracy less than $95 \%$ of the 5 -fold crossvaluation at each individual DNA variant, $\mathrm{MAF}<0.5 \%$, and deviation from HWE at $P$-value $<10^{-5}$, leaving 7 , 853,211 DNA variants for GWAS. With this WGS DNA panel, we demonstrated that the additive genetic variance and corresponding heritability estimates increased 


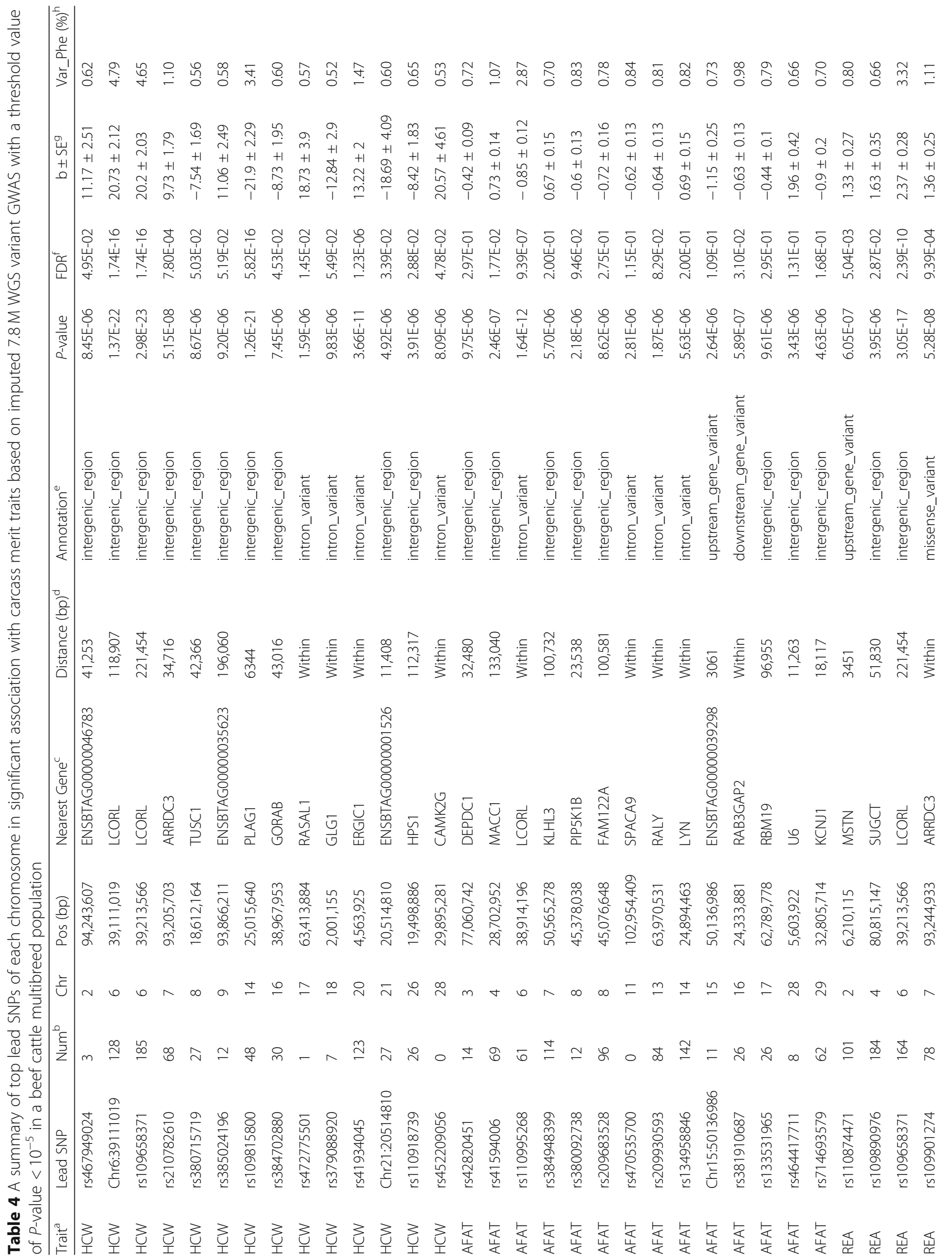




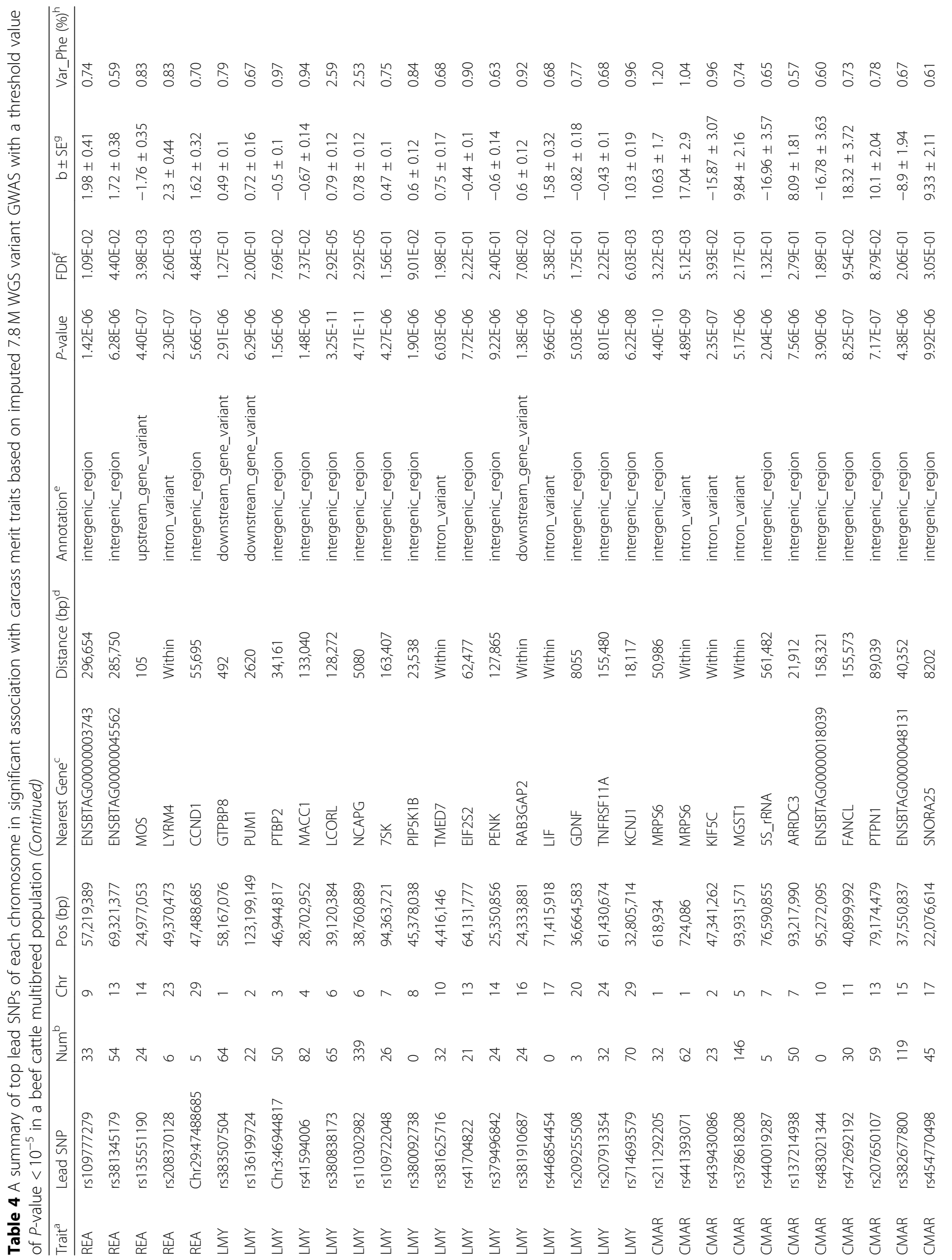




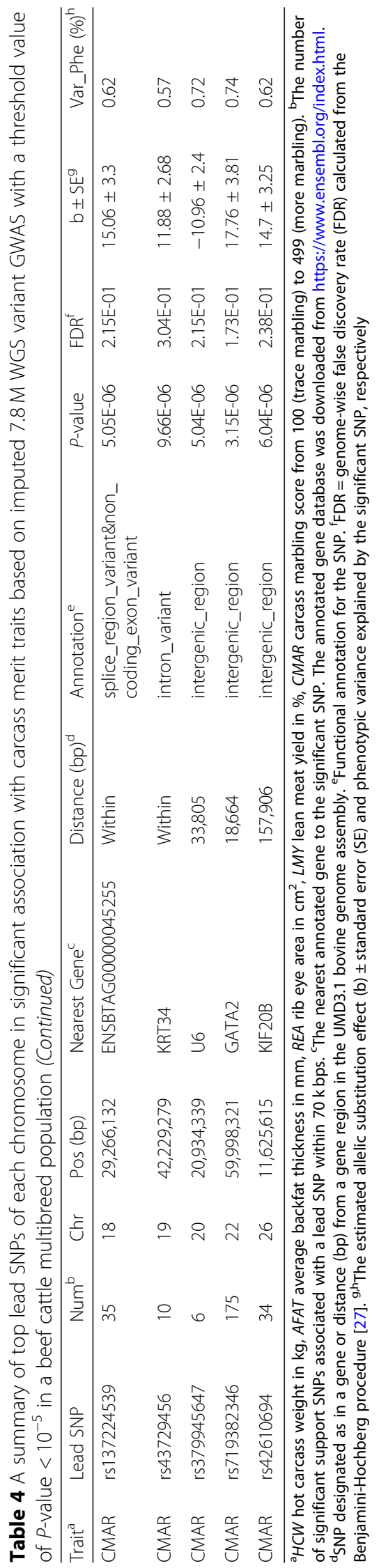


Table 5 Five topmost significantly enriched biological functions for carcass merit traits, and genes involved in the specific function

\begin{tabular}{|c|c|c|}
\hline Trait $^{\mathrm{a}}$ & Biological Function & Genes Involved in the biological function \\
\hline \multirow[t]{4}{*}{ HCW } & Gene expression (23) & $\begin{array}{l}\text { BMP7, BTRC, CTCFL, DTX1, HIF3A, IRF9, KAT7, KDM8, LGALS1, MAPK1, MRAS, MS4A15, NFIA, NR5A1, PARD3, PCTP, } \\
\text { PEG10, PPARGCIA, RNF4, RXRB, SIAH1, TADA3, TFCP2L1 }\end{array}$ \\
\hline & $\begin{array}{l}\text { Carbohydrate metabolism } \\
\text { (26) }\end{array}$ & $\begin{array}{l}\text { AGMO, ALPI, BID, BMP7, CMAS, CYP2J2, FCGR2B, GRPR, KDM8, LGALS1, MAPK1, MRAS, NGFR, PARD3, PCTP, PDK2, } \\
\text { PIGP, PIK3CB, PLA2G2A, PLEKHA3, PPARGCIA, PRKCB, PTHLH, ST8SIA1, UGT2B17, VDAC1 }\end{array}$ \\
\hline & Nucleic acid metabolism (18) & $\begin{array}{l}\text { ADCY4, ATP5PF, BMP7, CFTR, CMAS, GART, GNAI1, GRPR, MAPK1, NUDT9, OLA1, PDK2, PPARGC1A, PRKCB, PTHLH, } \\
\text { SLC25A5, ST8SIA1, VDAC1 }\end{array}$ \\
\hline & $\begin{array}{l}\text { Small molecule biochemistry } \\
(54)\end{array}$ & $\begin{array}{l}\text { ACSL6, AGMO, AKR1C3, AKR1C4, ALPI, ANGPTL4, ATP5PF, BID, BMP7, CFTR, CLEC11A, CMAS, CNTFR, CYP2J2, DHRS4, } \\
\text { ELOVL4, ERLIN1, FCGR2B, GBA3, GNAI1, GRPR, INHA, KCNE2, KCNE1B, LGALS1, MAPK1, MOGAT2, MRAS, NGFR, NR5A1, } \\
\text { P2RY13, PARD3, PCCB, PCSK2, PCTP, PDK2, PIGP, PIK3CB, PLA2G2A, PLEKHA3, PPARGC1A, PPARGC1B, PRKCB, PTHLH, } \\
\text { RXRB, SLC22A6, ST8SIA1 } \\
\text { TFCPLL1, TGM1, TTR, UGT2B11, UGT2B17, UPK2, VDAC1 }\end{array}$ \\
\hline
\end{tabular}

Molecular transport (45) ACSL6, AGMO, ALPI, ANGPTL4, ATP10A, ATP6V1E1, ATP6V1G1, BID, BMP7, CA4, CCS, CFTR, CLEC11A, CLIC4, CNTFR, COQ7, FCGR2B, GNAI1, GRPR, HBA1/HBA2, INHA, KCNE2, KCNE1B, KCNK2, LGALS1, MAPK1, MOGAT2, MRAS, NGFR, NR5A1, P2RY13, PCTP, PDK2, PIK3CB, PLA2G2A, PPARGC1A, PPARGC1B, PRKCB, PTHLH, SLC2OA2, SLC22A6, ST8SIA1, TTR, UPK2, VDAC1

AFAT Carbohydrate metabolism (22) AGMO, BID, BMP7, CMAS, GRPR, KDM8, LGALS1, MAPK1, MRAS, NGFR, PARD3, PDK2, PIK3CB, PLA2G2A, PPARGC1A, PPARGC1B, PTHLH, STRSIAT, UGT2B17

Nucleic acid metabolism (10) BID, BMP7, CMAS, GART, GNAI1, GRPR, MAPK1, PDK2, ST8SIA1, UGT2B17

Small molecule biochemistry (36)

ACSL6, AGMO, BID, BMP7, CFTR, CLEC11A, CMAS, CNTFR, DHRS4, ERLIN1, GART, GBA3, GNAI1, GRPR, KDM8, LGALS1, MAPK1, MOGAT2, MRAS, NGFR, NR5A1, P2RY13, PARD3, PDK2, PIGP, PIK3CB, PLA2G2A, PLEKHA3, PPARGC1A, PPARGC1B, PTHLH, SLC22A6, ST8SIA1, TFCP2L1, TGM1, UGT2B17

Lipid metabolism (31) ACSL6, AGMO, BID, BMPT, CFTR, CLECI1A, CNTFR, DHRS4, ERLIN1, GBA3, GNAI1, LGALS1, MAPK1, MOGAT2, MRAS, NGFR, NR5A1, P2RY13, PARD3, PDK2, PIGP, PIK3CB, PLA2G2A, PLEKHA3, PPARGC1A, PPARGC1B, PTHLH, ST8SIA1, TFCP2L1, TGM1, UGT2B17

Cell morphology (25) BID, BMP7, BTRC, CFTR, CLEC11A, CLIC4, CNTFR, FSCN1, GDF3, KCNK2, LGALS1, MAPK1, MRAS, NDUFAB1, NGFR, NR5A1, PCSK2, PLA2G2A, PLXNB2, PPARGC1A, PPARGC1B, PTHLH, SERPINA3, ST8SIA1, UPK2

REA Cell morphology (71)

BID, CAMP, CCND1, CD4, CERS5, CFTR, CHL1, CLEC11A, CLIC4, CNTFR, CSTB, CUL3, DVL1, EPO, FGL1, GDF3, GSDMD, HAND1, HAUS4, HELLS, INHA, INTU, KCNK2, KIF11, KIFC1, LGALS1, LIF, LIMK2, MAPK1, MAPT, NDUFAB1, NEFH, NFIA, NGFR, NTRK2, OSMR, P2RY12, PALLD, PCTP, PEG10, PLXNB2, PPARGC1A, PPARGC1B, PTHLH, PTPN1, RNF4, SCYL1, SERPINA3, UCP1, UPK2

Cellular assembly and organization (58)

AMPH, ARHGAP32, ARPC4, ATG4B, ATG4C, ATL1, BID, CAMP, CBLB, CCND1, CD4, CFTR, CLEC11A, CLIC4, CLTB, CSTB, CTDNEP1, DRP2, DVL1, EPO, EXO5, HAND1, IDE, KCNK2, KIF11, KIF13B, KIFC1, KLHDC8B, LANCL1, LGALS1, LIF, MAPT, NDUFAB1, NDUFS2, NEFH, NFIA, NGFR, NLGN1, NR5A1, NTRK2, OLA1, P2RY12, PALLD, PARD3, PLXNB2, POLG, PPARGC1A, PPARGC1B, REPS2, SERPINA3, SLC25A5, SNX9, SRCIN1, TP53INP1, TRAK2, TTR, UCP1, VDAC1

Cellular function and maintenance (51)

ARHGAP32, ARMC4, ATL1, BID, CAMP, CCDC103, CCDC39, CCND1, CD4, CELSR2, CLEC11A, CLIC4, COQ7, CSTB, DVL1, EPO, FCGR2B, HAND1, IDE, IFNA2, KCNK2, KIF11, KIF13B, KIFC1, LANCL1, LGALS1, LIF, MAPT, NDUFAB1, NDUFS2, NEFH, NFIA, NGFR, NLGN1, NMNAT3, NTRK2, PARD3, PLXNB2, POLG, PPARGCIA, PPARGCIB, SCYL1, SERPINA3, SS18, ST8SIA1, TCF7L1, TFCP2L1, TP53INP1, TRAK2, UCP1, VDAC1

Lipid metabolism (77) ABHD3, ACSL6, AGMO, AKR1C3, AKR1C4, AKR1C1/AKR1C2, ALPI, ANGPTL4, ANGPTL6, ATP5PF, BID, BMP7, C3AR1, CAMP, CD4, CERS5, CFTR, CLDN16, CLEC11A, CNTFR, CTDNEP1, CYP2C18, CYP2J2, CYP7B1, DEGS2, DHRS4, ELOVL4, EPO, ERLIN1, FCGR2B, FGL1, GBA3, GNAI1, GPC3, ILIRN, INHA, KCNE1B, KIF13B, KLF15, LGALS1, LIF, MAPK1, MAPT, MOGAT2, MRAS, NGFR, NONO, NR5A1, NTRK2, OSMR, P2RY12, P2RY13, PARD3, PCTP, PDK2, PIGP, PIK3CB, PLA2G2A, PLEKHA3, POLG, PPARGC1A, PPARGC1B, PRKCB, PTHLH, PTPN1, RENBP, RGS2, RXRB, SERPINE2, ST8SIA1, TFCP2L1, TRHR, TTR, UCP1, UGT2BA, UGT2B11, UGT2B17

Molecular transport (105)

ACSL6, AGMO, AKR1C4, AKR1C1/AKR1C2, ALPI, ANGPTL4, ANGPTL6, AOC3, APPBP2, ATP10A, ATP6V1E1, ATP6V1G1, BID, BMP7, C3AR1, CA4, CAMP, CBLB, CCS, CD4, CERS5, CFTR, CLDN16, CLEC11A, CLIC4, CNTFR, COQ7, CTDNEP1, DIO3, DUOXA2, DVL1, ELOVL4, EPO, FCGR2B, FGL1, GCNT4, GNAI1, GPC3, GRPR, HBA1/HBA2, ILIRN, INHA, IPGK1, KCNAB1, KCNE2, KCNE1B, KCNK2, KDM8, KIF13A, KIF13B, KLF15, LGALS1, LIF, MAPK1, MAPT, MOGAT2, MRAS, NDC1, NGFR, NONO, NR5A1, NTRK2, OGG1, OSMR, P2RY12, P2RY13, PCSK2, PCTP, PDK2, PIK3CB, PKN1, PLA2G2A, POLG, PPARGC1A, PPARGC1B, PRKCB, PTGER1, PTHLH, PTPN1, RENBP, RXRB, SCN9A, SLC16A4, SLC2OA2, SLC22A6, SLC37A2, SLC39A7, SLC6A7, SLC8B1, SMG6, SNX9, SRCIN1, ST8SIA1, STRADA, STRADB, SVBP, SYNDIG1, TMED2, TP53INP1, TRAK2, TRHR, TTR, UCP1, VDAC1, ZFP36L1

LMY Gene expression (23) BMP7, BTRC, CTCFL, DTX1, HIF3A, IRF9, KAT7, KDM8, LGALS1, MAPK1, MRAS, MS4A15, NFIA, NR5A1, PARD3, PCTP, PEG10, PPARGC1A, RNF4, RXRB, SIAH1, TADA3, TFCP2L1

Lipid metabolism (47)

ACSL6, AGMO, AKR1C3, AKR1C4, ALPI, ANGPTL4, ATP5PF, BID, BMP7, CFTR, CLEC11A, CNTFR, CYP2J2, DHRS4, ELOVL4, ERLIN1, FCGR2B, GBA3, GNAI1, INHA, KCNE1B, LGALS1, LIF, MAPK1, MOGAT2, MRAS, NGFR, NRSA1, P2RY13, PARD3, PCCB, PCTP, PDK2, PIGP, PIK3CB, PLA2G $2 A$, PLEKHA3, PPARGCIA, PPARGCIB, PRKCB, PTHLH, RXRB, ST8SIA1, TFCP2LI, TTR, UGT2B11, UGT2B17 
Table 5 Five topmost significantly enriched biological functions for carcass merit traits, and genes involved in the specific function (Continued)

\begin{tabular}{|c|c|c|}
\hline Trait $^{\mathrm{a}}$ & Biological Function & Genes Involved in the biological function \\
\hline & $\begin{array}{l}\text { Small molecule biochemistry } \\
\text { (55) }\end{array}$ & $\begin{array}{l}\text { ACSL6, AGMO, AKR1C3, AKR1C4, ALPI, ANGPTL4, ATP5PF, BID, BMP7, CFTR, CLEC11A, CMAS, CNTFR, CYP2J2, DHRS4, } \\
\text { ELOVL4, ERLIN1, FCGR2B, GBA3, GNAI1, GRPR, INHA, KCNE1B, KCNE2, LGALS1, LIF, MAPK1, MOGAT2, MRAS, NGFR, } \\
\text { NR5A1, P2RY13, PARD3, PCCB, PCSK2, PCTP, PDK2, PIGP, PIK3CB, PLA2G2A, PLEKHA3, PPARGC1A, PPARGC1B, PRKCB, } \\
\text { PTHLH, RXRB, SLC22A6, ST8SIA1, TFCP2L1, TGM1, TTR, UGT2B11, UGT2B17, UPK2, VDAC1 }\end{array}$ \\
\hline & $\begin{array}{l}\text { Vitamin and mineral } \\
\text { metabolism (17) }\end{array}$ & $\begin{array}{l}\text { AKR1C3, AKR1C4, BMP7, CFTR, CYP2J2, DHRS4, INHA, LIF, NR5A1, P2RY13, PCTP, PPARGC1A, ST8SIA1, TTR, UGT2B11, } \\
\text { UGT2B17, VDAC1 }\end{array}$ \\
\hline & $\begin{array}{l}\text { Carbohydrate metabolism } \\
\text { (26) }\end{array}$ & $\begin{array}{l}\text { AGMO, ALPI, BID, BMP7, CMAS, CYP2J2, FCGR2B, GRPR, KDM8, LGALS1, MAPK1, MRAS, NGFR, PARD3, PCTP, PDK2, } \\
\text { PIGP, PIK3CB, PLA2G2A, PLEKHA3, PPARGC1A, PRKCB, PTHLH, ST8SIA1, UGT2B17, VDAC1 }\end{array}$ \\
\hline \multirow[t]{5}{*}{ CMAR } & $\begin{array}{l}\text { Carbohydrate Metabolism } \\
\text { (23) }\end{array}$ & $\begin{array}{l}\text { AGMO, BID, BMP7, CMAS, GNAI1, GRPR, KDM8, LGALS1, MAPK1, MRAS, NGFR, PARD3, PCTP, PDK2, PIGP, PIK3CB, } \\
\text { PLA2G2A, PLEKHA3, PPARGC1A, PPARGC1B, PTHLH, ST8SIA1, UGT2B17 }\end{array}$ \\
\hline & Nucleic acid metabolism (10) & BID, BMP7, CMAS, GART, GNAI1, GRPR, MAPK1, PDK2, ST8SIA1, UGT2B17 \\
\hline & $\begin{array}{l}\text { Small molecule biochemistry } \\
(40)\end{array}$ & $\begin{array}{l}\text { ACSL6, AGMO, AKR1C3, AKR1C4, BID, BMP7, CFTR, CLEC11A, CMAS, CNTFR, DHRS4, ERLIN1, GART, GBA3, GNAI1, } \\
\text { GRPR, KDM8, LGALS1, MAPK1, MOGAT2, MRAS, NGFR, NR5A1, P2RY13, PARD3, PCSK2, PCTP, PDK2, PIGP, PIK3CB, } \\
\text { PLA2G2A, PLEKHA3, PPARGC1A, PPARGC1B, PTHLH, SLC22A6, ST8SIA1, TFCP2L1, TGM1, UGT2B17 }\end{array}$ \\
\hline & Cellular development (24) & $\begin{array}{l}\text { AKR1C3, B9D1, BID, BMP7, CBLB, CLEC11A, CLIC4, FSCN1, ITGA11, ITIH4, KCNK2, LGALS1, MAPK1, MRAS, NASP, NGFR, } \\
\text { NRSA1, PIK3CB, PPARGC1A, PTHLH, TGM1, UGT2B17, UPK2, ZFP36L1 }\end{array}$ \\
\hline & Lipid metabolism (33) & $\begin{array}{l}\text { ACSL6, AGMO, AKR1C3, AKR1C4, BID, BMP7, CFTR, CLEC11A, CNTFR, DHRS4, ERLIN1, GBA3, GNAI1, LGALS1, MAPK1, } \\
\text { MOGAT2, MRAS, NGFR, NR5A1, P2RY13, PARD3, PCTP, PDK2, PIGP, PIK3CB, PLA2G2A, PLEKHA3, PPARGC1A, } \\
\text { PPARGC1B, PTHLH, ST8SIA1, TFCP2L1, UGT2B17 }\end{array}$ \\
\hline
\end{tabular}

${ }^{\mathrm{a}} \mathrm{HCW}$ hot carcass weight in $\mathrm{kg}, A F A T$ average backfat thickness in $\mathrm{mm}, R E A$ rib eye area in $\mathrm{cm}^{2}, L M Y$ lean meat yield in \%, CMAR carcass marbling score from 100 (trace marbling) to 499 (more marbling)

by 6.1 to $22.5 \%$ for all the five carcass traits in comparison to the $50 \mathrm{~K}$ SNP panel (Table 1). Moreover, the 7 , 853,211 DNA variant based GWAS identified additional significant DNA variants for all five carcass merit traits in comparison to the $50 \mathrm{~K}$ SNPs. In a companion study, we also observed that the imputed 7.8 M DNA variants accounted for more additive genetic variance and led to identification of additional DNA variants that are associated with feed efficiency and growth traits in beef cattle [23], indicating that the imputed 7.8 M WGS variants can improve the power of GWAS analyses for beef cattle quantitative traits.

\section{DNA marker effect distributions}

The distributions of DNA marker allele substitution effects and the amounts of additive genetic variances explained by single DNA markers support the assumptions of a normal distribution for SNP effects and a scaled inverse-chi squared distribution for SNP additive genetic variance used in previous studies [32, 33], although these DNA marker effect distributions may be biased as greater LD between DNA markers in the $7.8 \mathrm{M}$ DNA variant panel is expected and a single DNA marker GWAS was used in this study. The 7.8 M DNA variant GWAS also demonstrated that the majority of the variants had zero or near zero effects on all the five carcass merit traits, and only a small fraction $(<0.1 \%)$ of the 7,853,211 WGS variants passed the suggestive threshold of $P$-value $<0.005$. This seems to correspond well to a $\pi$ value of approximately $99 \%$ that was commonly used as an assumption to shrink proportions of
SNPs to no effects in genetic analyses with high density SNPs [34]. Another important aspect of quantitative trait genetic architecture is whether the trait is affected by many genes with small effects or by a few of genes with large and/or modest effects plus genes with small effects. The GWAS results based on the 7.8 M DNA variants showed that HCW, LMY, and REA are likely influenced by a few of genes with larger effects, explaining up to $4.79 \%$ phenotypic variance, and many genes with small effects. However, for AFAT and CMAR, a few of genes with modest effects and many genes with small effects likely contribute to the variation of the traits (Table 4).

\section{DNA marker effects related to SNP functional classes}

Annotation of DNA variants into functional classes allows for further dissection of DNA marker effects on the trait to DNA variant functionality. The imputed 7.8 M DNA variants include a proportionally larger number of DNA polymorphisms in each of the functional classes, ranging from 3309 for 5'UTR variants to $5,251,680$ for intergenic region in comparison to the lower density SNP panels such as the bovine $50 \mathrm{~K}$ SNPs, which was reported in the Additional files of Zhang et al. (2019) [23]. For convenience, the annotation information of various DNA variant panels has been provided in Additional file 3: Tables S1-S3 of this article. We used both the average squared allele substitution effects of each functional class and the additive genetic variance captured by a single DNA variant within the functional class to assess their relative importance in affecting the trait. For the 
average squared allele substitution effects, missense variants, 3'UTR, 5'UTR, and other regulatory region variants exhibited a relatively larger allele substitution effect on all five carcass merit traits in general in comparison to variants in other functional classes. Although the LD between DNA markers of different functional classes and the singe DNA marker GWAS used in this study may lead to biased estimates of the DNA marker effect on the traits, the results are in agreement with the expectation that missense variants alter the peptide sequence of a protein, and greater roles of 3'UTR, 5'UTR, and other regulatory variants play in influencing gene expression and gene translation [35-37].

To provide further insight into relative importance of each functional class, we fitted the GRM of the functional class and GRM constructed from DNA variants of all other functional classes simultaneously to estimate the additive genetic variance captured by each functional class. For each functional class, the sum of the additive genetic variances captured by the two GRMs (Table 3) was almost the same as the additive genetic variance obtained by the GRM with all the imputed 7.8 M DNA variants for all the traits (Table 1), indicating a reliable partition of additive genetic variance for each function class variants for the carcass merit traits. Although intergenic variants and intron variants captured a greater amount of total additive genetic variance for all five carcass merit traits, their relative proportion of additive genetic variance explained per sequence variant was smaller than other functional classes. These results concur with the report by Koufariotis et al. [38] that the intron and intergenic variants explained the lowest proportion of the genetic variance per SNP for milk and fertility traits in dairy cattle. Relatively smaller amount of additive genetic variance captured per sequence variant in intron and intergenic regions were also observed for feed efficiency related traits in beef cattle [23], which is likely due to much larger numbers of DNA variants in the class and the majority of them have small or zero effects on the traits. Of other functional classes, 3'UTR explained more additive genetic variance per DNA variant for HCW, AFAT, and REA while DNA variants in 5'UTR and other regulatory variants also showed a greater amount of additive genetic variance explained per sequence variant for CMAR and for CMAR and REA, respectively. It was found that missense variants captured a greater amount of additive genetic variance per sequence variant for REA, LMY, and CMAR. Although synonymous variants had relatively small average squared SNP allele substitution effects, a single DNA variant in the functional class accounted for more additive genetic variance for AFAT, REA, LMY, and CMAR. In addition, both the downstream and upstream gene variants were found to capture more additive genetic variance per sequence variant for HCW (Table 3). These results suggest that the relative contribution per DNA variant of the functional classes to the additive genetic variance might vary across different traits. Indeed, in a study by Koufariotis et al. [39], functional classes including splice sites, 3'UTR, 5'UTR, and synonymous variants explained relatively a larger proportion of genetic variance per sequence variant for milk production traits but not for fertility related traits.

It was observed that most top lead SNPs with larger effects are located between genes or located in intronic regions, although their average SNP effects or variances captured by individual DNA variants were relatively smaller than missense and regulatory DNA variants including 3'UTR and 5'UTR variants (Table 4). However, there were cases for each trait where support SNPs had either larger allele substitution effects or explained a larger percentage of phenotypic variance than those of their lead SNPs but with a larger $P$-value. For instance, a missense variant rs42661323 at 4,916,731 bp on BTA20 had an allele substitution effect of 10.73 on $\mathrm{HCW}$, which was larger than that $(b=10.14)$ of its nearby lead SNP rs41574252 located at 4863507 bp. However, the $P$-value of the missense variant $r s 42661323$ was $8.10 \times 10^{-8}$ and was slightly larger than that $\left(P\right.$-value $=4.85 \times 10^{-8}$, or 4.85E-08) of its lead SNP (Additional file 2). A similar instance was found for missense variant $r s 379314731$ of gene ENSBTAG00000012585 (RAB3GAP2) at 24,332, $917 \mathrm{bp}$ on BTA16 for AFAT. The missense variant rs379314731 had an allele substitution effect of -0.64 on AFAT with a $P$-value of $8.76 \times 10^{-7}$. However, its nearby downstream gene SNP rs381910687 was selected as the lead SNP due to its lower $P$-value of $5.89 \times 10^{-7}$ although its allele substitution effect was slightly smaller (i.e. $b=-0.63$ ). Therefore, support SNPs that are located in more important functional classes such as missense and regulatory variants are also worth further investigation. Additionally, as the imputed $7.8 \mathrm{M}$ DNA variants represent a proportion of whole genome DNA polymorphisms, the intergenic or intronic SNPs with larger effects may also be in LD with the causative DNA variant(s) that are not present in the 7.8 M DNA variant panel. In this case, fine mapping of QTL in the region of lead SNPs is needed to identify the causative DNA variants for the trait.

\section{QTLs for carcass merit trait in beef cattle}

Mapping QTLs via linkage or association analyses are subject to a false positive rate. Therefore, validation of QTL or DNA variants associated with a trait in independent studies provides confidence on the identified candidate QTLs or DNA variants. We compared our lead significant SNPs with the QTL regions reported in 
the Cattle QTL database (https://www.animalgenome. org/cgi-bin/QTLdb/index, accessed on 22 August 2018) [14]. With a window centered at the lead SNPs extending $70 \mathrm{~kb}$ upstream and downstream, 33, 17, 20, 3, and 0 were overlapped with reported QTL for HCW, AFAT, REA, LMY, and CMAR, respectively (Additional file 3: Table S4). With a window of $1 \mathrm{Mb}, 41$ of the 51 lead SNPs for HCW, 20 of the 33 lead SNPs for AFAT, 31 of the 46 lead SNPs for REA, 15 of the 40 lead SNPs for LMY, and 2 of the 39 lead SNPs for CMAR were found to be overlapped with the reported QTL in the Cattle QTL database (Additional file 3: Table S4). These overlapped lead SNPs provide additional evidence that the QTL regions may harbor causative DNA variants affecting the carcass merit traits. The non-overlapped lead SNPs, however, may suggest unique QTLs that were segregating in the investigated beef cattle population for the trait, in particular for the lead SNPs with multiple support SNPs (Table 4).

To investigate potential pleotropic effects of SNPs or QTL regions on the carcass merit traits, we also compared lead significant SNPs among the five carcass merit traits. It was found that CMAR did not share any lead significant SNPs with HCW, AFAT, REA, or LMY. HCW, AFAT, REA, and LMY, however, shared a common significant lead SNP "rs109696064", which was a downstream gene variant that is 3164 bps away from the nearest gene LCORL on chromosome 6 (Additional file 2). AFAT and HCW also shared four lead significant SNPs located on chromosome 6, including one intronic variant (rs109355965) that is within gene ENSBTAG00000005932 (FAM184B), one intronic variant (rs110995268) of gene LCORL, one downstream gene variant (rs109843602) that was in proximity to genes NCAPG and DCAF16, and one downstream gene variant (rs109696064) located within $70 \mathrm{~kb}$ of genes LCORL and NCAPG. The region that harbors genes NCAPG-LCORL on BTA6 is likely to be a candidate QTL region with pleiotropic effects for carcass merit traits including HCW, AFAT, REA, and LMY. The lead significant SNPs located on BTA 6 in the region of $37.9 \mathrm{Mb}$ to $39.9 \mathrm{Mb}$ were also found to have relatively larger effects on HCW, AFAT, REA, and LMY as shown in the Manhattan plots (Fig. 1). The chromosome region (i.e 6_37 to 6_39) was previously reported to have large pleiotropic effects on traits including carcass weight, rib eye muscle area, and carcass fat thickness in multiple US cattle breeds [40]. In our 7.8 M DNA variant GWAS for feed efficiency related traits, this chromosomal region also showed the largest effects on DMI, ADG, and MWT, explaining from 3.04 to $5.80 \%$ phenotypic variance for the traits as reported by Zhang et al. in our companion paper [23]. All these results strengthen the evidence that there are likely causative DNA variants in the chromosomal region with major pleiotropic effects on beef cattle growth related traits [40]. Genes NCAPG and LCORL are the two major nearest genes to the chromosomal region. DNA markers within or in proximity to genes NCAPG (Non-SMC Condensin I Complex Subunit) and LCORL (ligand-dependent nuclear receptor co-repressor like) were found to have significant associations with feed intake and body weight gain in beef cattle [41]. In our study, the annotation of the imputed 7.8 M DNA marker panel identified a total of 185 WGS variants within NCAPG including 4 synonymous variants, 177 intronic variants, 2 missense variants, and 2 other regulatory region variants. Also a total of 409 WGS variants were within gene $L C O R L$, including 404 intronic variants, 1 missense variant, and 4 3'UTR variants. At $P$-value less than $10^{-5}, 17$ SNPs (including 15 intronic variants and 2 missense variants) within gene NCAPG were found to be in significant association with $\mathrm{HCW}$ but none of them were identified to be a lead SNP. The intronic SNP rs110175987 of NCAPG was significantly associated with HCW (i.e. AC_000163.1:g.38783305C $>$ T, $P$-value $=$ $1.14 \times 10^{-19}$ and $\mathrm{FDR}=1.51 \times 10^{-15}$ ), accounting for $4.18 \%$ of the phenotypic variance, and it was the largest proportion of phenotypic variance explained by a single DNA marker among the 17 within-gene variants (Additional file 2). This SNP was also significantly associated with AFAT $\left(P\right.$-value $=5.42 \times 10^{-12}$ and FDR $\left.=1.33 \times 10^{-6}\right)$, REA $\left(P\right.$-value $=1.34 \times 10^{-15}$ and FDR $\left.=3.99 \times 10^{-10}\right)$ and LMY $\left(P\right.$-value $=1.20 \times 10^{-10}$ and FDR $\left.=2.92 \times 10^{-5}\right)$, explaining $2.72,3.19$, and $2.41 \%$ of the phenotypic variance, respectively (Additional file 2). A missense variant rs 109570900 at $38,777,311 \mathrm{bp}$ on BTA6, which induces a Ile-442-Met substitution in amino acid within NCAPG, was also identified to be in significant association with $\mathrm{HCW}$ $\left(P\right.$-value $=2.10 \times 10^{-9}$ and FDR $\left.=4.65 \times 10^{-5}\right)$ and REA $\left(P\right.$-value $=5.09 \times 10^{-8}$ and FDR $\left.=9.39 \times 10^{-4}\right)$ accounting for 1.45 and $1.18 \%$ of phenotypic variance, respectively. Previous studies reported that this missense variant had strong association with fetal growth and birth weight in Holstein and Charolais crossbreed [42]. The missense variant was also in association with body frame size at puberty in Japanese black and Charolais $\times$ Holstein [43] and with carcass weight, longissimus muscle area, and subcutaneous fat thickness in Japanese Black and Brown cattle [44]. Sahana et al. [45] proposed the missense as a strong candidate responsible for calf size at birth and consequently calf birth survival. In our companion paper by Zhang et al. [23], the SNP within NCAPG was also found to be associated with ADG, DMI, and MWT, respectively.

For gene LCORL, the intronic SNP rs110995268 at $38,914,196$ bp was significantly associated with AFAT $\left(P\right.$-value $=1.64 \times 10^{-12}$ and FDR $\left.=9.39 \times 10^{-7}\right)$, explaining $2.87 \%$ of the phenotypic variance (Table 4$)$. The SNP was also significantly associated with $\mathrm{HCW}(P$-value $=$ $4.2 \times 10^{-20}$ and $\left.\mathrm{FDR}=6.76 \times 10^{-15}\right)$, REA $(P$-value $=$ 
$1.80 \times 10^{-15}$ and FDR $\left.=4.12 \times 10^{-10}\right)$, and LMY $(P$-value $=$ $6.15 \times 10^{-11}$ and $\mathrm{FDR}=2.92 \times 10^{-5}$ ), explaining 4.33, 3.19 , and $2.50 \%$ of the phenotypic variance, respectively (Additional file 2). A total of 80, 15, 47, and 15 SNPs within gene $L C O R L$ were identified to be significantly associated with HCW, AFAT, REA, and LMY respectively. However, they were all intronic variants. The intronic SNP rs 110995268 belongs to a group of 15 common significant intronic variants within $L C O R L$ that had effects on HCW, AFAT, REA, and LMY. Out of the 15 withingene intronic SNPs, proportions of phenotypic variance ranged from 4.30 to $4.37 \%$ for $\mathrm{HCW}$, from 2.82 to $2.87 \%$ for AFAT, from 3.10 to $3.24 \%$ for REA, and 2.44 to $2.50 \%$ for LMY (Additional file 2).

The NCAPG-LCORL region also encompassed two additional interesting genes including DCAF16 and FAM184B. In addition, gene SNPs under other lead significant QTL regions with relatively larger effects were also examined and some significant lead SNPs were found to be missense or located within regulatory regions (Table 4), which may suggest their roles as causative mutations due to the functional annotation. For instance, a missense variant rs109901274 within gene ENSBTAG00000007116 (ARRDC3) at 93,244,933 bp on chromosome 7 was a lead SNP in significant association $\left(P\right.$-value $\left.=5.28 \times 10^{-8}\right)$ with REA, explaining $1.11 \%$ of phenotypic variance (Table 4). The SNP rs109901274 was also found to be a significant support SNP in association with $\mathrm{HCW}$, with a $P$-value of $8.84 \times 10^{-8}$ and accounted for $1.07 \%$ of phenotypic variance (Additional file 2). Gene ARRDC3, which harbours SNP rs109901274, belongs to an arrestin superfamily and plays a role in regulating body mass in mice [46] and human males [47]. In our companion paper by Zhang et al. [23], SNP rs109901274 was also reported to be a lead SNP in significant association with ADG and MWT. A previously study by Saatchi et al. reported that SNPs in proximity to $A R R D C 3$ were associated with birth weight, carcass weights, and body weights in US cattle breeds [40]. However, the physiological roles of $A R R D C 3$ in cattle remain unknown.

It was commonly observed that SNPs from the intronic region of the genes or between genes showed significant effects on the carcass merit traits as lead SNPs. For instance, one of the most significant lead SNPs (rs109815800, AC_000171.1:g.25015640G $>\mathrm{T}, P$-value $=$ $1.26 \times 10^{-21}$ and FDR $=5.82 \times 10^{-16}$ ) in association with $\mathrm{HCW}$ on chromosome 14 at $25015,640 \mathrm{bp}$ was mapped to the intergenic region (6344 bp upstream) of PLAG1 (Table 4). This SNP was previously reported as one of the eight candidate QTNs with major effects on bovine stature by Karim et al. [48]. The SNP was also the most significant DNA marker reported by Fink et al. [49] in expression QTL mapping of PLAG1, and the most significant SNP in meta-analysis of GWAS for cattle stature by Bouwman et al. [50]. This SNP (i.e. rs 109815800) accounted for $3.41 \%$ of phenotypic variance of HCW in this study (Table 4). Additionally, SNP rs109815800 was a support SNP in significant association with REA, reaching a $P$-value of $2.02 \times 10^{-6}$ and explained $0.84 \%$ of phenotypic variance (Additional file 2). These intronic DNA variants significantly associated with the traits may also warrant further investigation for their effects on the traits. In addition, the significant intronic and intergenic DNA variants may also in high LD with the causative DNA variant(s) that are not present in the imputed 7.8 M DNA variant panel. Therefore, further fine mapping of the QTL regions will lead to identification of causative variants for the carcass merit traits in cattle, in particular for QTL regions where lead SNPs are supported by multiple significant DNA markers.

\section{Genetic networks compared with RNAseq}

The IPA analyses based on the candidate genes identified via a window of $70 \mathrm{kbp}$ of the lead SNPs with FDR < 0.10 detected lipid metabolism was among the top 5 enriched molecular process for four of the carcass merit traits (AFAT, CMAR, LMY, and REA), and 6th for $\mathrm{HCW}$, followed by carbohydrate metabolisms and small molecule biochemistry. In studies using RNAseq on bovine liver samples, lipid metabolism, and small molecule biochemistry were also among the top enriched molecular processes for marbling score in Charolais steers [51, 52]. In this study, all the animals with carcass data were finished for meat production. The goal of the fattening stage with a finishing diet is to allow beef cattle to grow muscle and to accumulate intramuscular fat, i.e. marbling, for better carcass quality. Therefore, genes involved in lipid metabolism and carbohydrate metabolism likely play a more important role in determining the carcass merit traits, as shown both in this and previous studies $[51,52]$. The identification of top and other enriched molecular processes and their corresponding genes will not only improve our understanding on genetic mechanisms that influence the carcass traits but also help prioritize candidate genes for identification of causative gene polymorphisms responsible for the phenotypic variation.

\section{Conclusions}

The imputed 7,853,211 DNA variants explained more genetic variance than the $50 \mathrm{~K}$ SNP panel and led to identification of additional QTL regions in associations with carcass merit traits in Canadian multi-breed beef cattle. The DNA marker allele substitution effects on the carcass traits based on the imputed 7,853,211 DNA variants approximated a bell-shaped distribution, and the additive genetic variances explained by single DNA 
variants followed a scaled inverse chi-squared distribution to a greater extent. On average, missense variants, 3'UTR variants, 5'UTR variants, and other regulatory region variants exhibited larger allele substitution effects in comparison to DNA variants that are located between genes and in intronic regions. Intergenic and intronic variants also accounted for a smaller amount of additive genetic variance per DNA variant for the carcass traits whereas single regulatory, synonymous, and missense variants had relatively larger impacts on the variation of carcass merit traits. The five carcass merit traits appear to be controlled by a few DNA variants with relatively larger or modest effects complementary by DNA variants with small effects. Lipid metabolism, small molecular biochemistry, and carbohydrate metabolism were the top biological processes for the carcass merit traits. The genetic architecture as revealed by the $7.8 \mathrm{M}$ DNA variant GWAS will improve our understanding on the genetic control of carcass merit traits in beef cattle.

\section{Methods}

\section{Animal populations and phenotype data}

The populations used in this study, i.e., Angus, Charolais, Kinsella Composite, Elora crossbred, PG1, and TXX, were described previously [23, 53-56]. Briefly, Angus, Charolais, and Kinsella Composite herds are located at Roy Berg Kinsella Research Ranch, University of Alberta, with Angus and Charolais being maintained as purebreds while the Kinsella Composite herd had been influenced mainly by Angus, Charolais, Galloway, and Hereford. The Elora crossbred animals were from the Elora Beef Research Centre, University of Guelph and it was made by crossing Angus, Simmental, Charolais, and other cattle breeds. Both the commercial crossbred PG1 and terminal crossbred TXX animals were from multiple commercial herds in Alberta. The top beef breeds that were used in commercial crossbred beef production in Alberta included Angus, Charolais, Herefore, Simmental, Limousin, Gelbvieh, while the TXX animals were produced from 2- or 3-way crossbreeding systems involving terminal composite bulls (TX/TXX) and crossbred cows of multiple beef breeds. Animals used in this study were finishing steers and heifers born between 1998 and 2006 for the Elora crossbred, between 2002 and 2015 for Kinsella Composite, between 2004 and 2015 for Angus and Charolais, between 2008 and 2011 for PG1 and TXX populations.

The animals were initially measured for feed intake using the GrowSafe system (GrowSafe Systems Ltd., Airdrie, Alberta, Canada) at their respective feedlot test station under multiple projects, which were described previously [55, 57-59]. After the feedlot tests, animals were slaughtered either at a commercial plant or at the
Lacombe Research and Development Centre (LRDC) abattoir when a majority of them reached $>8 \mathrm{~mm}$ backfat thickness as predicted from ultrasound measurements. For slaughter, animals were first stunned by captive bolt and then exsanguinated. Collection of carcass data was previously described [53, 55, 59-62]. Briefly, hot carcass weight ( $\mathrm{HCW}$ ) in $\mathrm{kg}$ was obtained by summing up the weight of each side of the carcass that was split during dressing, about $45 \mathrm{~min}$ post-mortem. Average backfat thickness (AFAT) in mm, rib eye area (REA) in squared centimeters, and carcass marbling score (CMAR) at the grading site between the 12th and 13th ribs was assessed by trained personnel. Carcass marbling score was measured as a continuous variable from 100 (trace marbling or less) to 499 (abundant or more marbling) to reflect the amount of fat deposit interspersed between the muscle fibers (i.e., intramuscular fat) of the longissimus thoracis. Lean meat yield (LMY) was calculated as LMY, $\%=57.96+\left(0.202 \times\right.$ REA, $\left.\mathrm{cm}^{2}\right)$ $-(0.027 \times \mathrm{HCW}, \mathrm{kg})-(0.703 \times \mathrm{AFAT}, \mathrm{mm})$ as described by Basarab et al. [57] as an estimate of saleable meat in the carcass. The phenotype data obtained from each data source were examined and phenotypic values beyond 3 standard deviations of the trait value mean were excluded from further analyses.

\section{SNP data consolidation, imputation, and functional annotation}

All animals entering the feedlot tests were genotyped with bovine $50 \mathrm{~K}$ SNP panels under multiple projects. SNP data consolidation and imputation was described in the companion paper [23]. Briefly, raw $50 \mathrm{~K}$ SNP genotype profile data were obtained from each source and SNP genotypes were then called in each of the four different SNP formats, i.e. forward strand, top strand, design strand, and $\mathrm{AB}$ format. The SNP genotype data were then combined by the same SNP format and each SNP was examined to ensure it had only two alleles after merging. In total, $50 \mathrm{~K} \mathrm{SNP}$ genotypes of 11,448 beef cattle were compiled. A SNP quality check was applied for each data source, where SNPs that had a minor allele frequency less than $5 \%$, or had a missing rate larger than $5 \%$, or were significantly deviated from exact test of Hardy-Weinberg equilibrium (HWE) $\left(P\right.$-value $\left.<10^{-3}\right)$, or on sex chromosomes were filtered out. SNPs removed from one data source were also excluded from all other data sources. In addition, animals with more than a $5 \%$ missing rate of total SNP genotypes were deleted. After SNP data editing, 33,321 SNPs were retained for further analyses. Sporadic missing SNP genotypes in the SNP data set $(<0.065 \%)$ were then imputed via the population-based algorithm implemented in Beagle 3.3.2 [63]. Population admixture analyses were also conducted for all the 11,448 beef cattle based on the 33,321 SNPs 
to predict breed composition for each animal, which was described in the companion paper [23].

SNP imputation was conducted using FImpute 2.2 [28] in a two-step procedure: (1) from the $50 \mathrm{~K}$ SNPs (i.e. 33,321 SNPs) to the Affymetrix Axiom GenomeWide BOS 1 Array (Affymetrix, Inc., Santa Clara); (2) from imputed HD to the full whole-genome sequence (WGS) variants in run 5 of the 1000 Bull Genomes Project [22]. Details of SNP imputation and average imputation accuracy for each chromosome were provided in the companion paper [23]. Initially, 38,318,974 imputed WGS variant genotypes were obtained for all the animals. Quality control was then performed on the imputed WGS variant genotypes to ensure better quality of imputed genotype data, where DNA variant genotypes with less than 95\% imputation accuracy, or being homozygous, or with a minor allele frequency (MAF) less than 0.005 in either population/breed, or with significant deviations from Hardy-Weinberg exact test at significance levels of $P$-value $<10^{-5}$ in either population/breed were excluded from further analyses. The post-imputation quality control resulted in 7,853,211 DNA variant genotypes that contain 30,155 SNPs from the $50 \mathrm{~K}$ SNP genotypes on all the animals. The 7,853,211 DNA variants included 7,497,128 SNPs and 356,083 INDELs (termed 7.8 M DNA variants or 7.8 M DNA variant panel or 7.8 M SNP panel in the text). The imputed 30,155 SNPs in the 7.8 M DNA variant panel were replaced by their actual genotypes to facilitate comparison of the $50 \mathrm{~K} \mathrm{SNP}$ panel and the 7.8 M DNA variants panel.

Functional annotation of SNPs or DNA variants on the 30,155 SNPs and on the 7,853,211 DNA variants was provided in the companion paper [23]. The WGS DNA variants were annotated through run 5 of the 1000 Bull Genomes Project, which included 379 full genome sequences from the Canadian Cattle Genome Project [64]. DNA variants were then assigned to a functional class based on their overlap with gene features described in the Ensembl database (release 81), using an updated version of the NGS-SNP annotation system [65]. These SNPs were grouped into 9 broader functional classes, which consisted of intergenic region variants, downstream gene variants, upstream gene variants, synonymous variants, intron variants, missense variants, 3' UTR variants, $5^{\prime}$ UTR variants, and other regulatory region variants that includes splice regions in intron variants, disruptive in-frame deletion, and splice region variants, etc. (Additional file 3: Table S1-S3).

\section{Genome wide association analyses}

Animals with carcass data were merged with their imputed genotype data in the $7.8 \mathrm{M}$ DNA variant panel, resulting in a sample size of $n=3354$ for AFAT to $n=$ 3984 for HCW (Table 1). For the GWAS analyses, phenotypic values of the five carcass traits were adjusted for animal birth year, sex type, a combination of feedlot test location and pen, breed composition fraction of each postulated ancestral breed predicted using the $50 \mathrm{~K} \mathrm{SNP}$ panel and Admixture [66], and animal age at slaughter. The GWAS analyses were performed using a single SNP-based mixed linear model association (MLMA) as implemented in GCTA software [67,68], and the linear mixed model can be described as follows:

$$
y_{i j}=\mu+b_{j} x_{i j}+a_{i j}+e_{i j}
$$

where $y_{i j}$ is the adjusted phenotypic value of the ith animal with the jth SNP (i.e. the ijth animal, bj is the allele substitution effect of SNPj, $x_{i j}$ is the jth SNP genotype of animal i, and it was coded as 0, 1, 2 for genotypes $A_{1} A_{1}$, $A_{1} A_{2}$ and $A_{2} A_{2}$, respectively, $\mathrm{a}_{\mathrm{ij}}$ is the additive polygenic effect of the $i j$ th animal $\sim N\left(0, G \sigma_{a}^{2}\right)$, and $\mathrm{e}_{\mathrm{ij}}$ is the random residual effect $\sim N\left(0, \boldsymbol{I} \sigma_{e}^{2}\right)$. The genomic relationship matrix G (GRM) was constructed using GCTAGRM as implemented in GCTA software and defined in Yang et al. [67, 69], which is essentially the same as the G matrix calculated by the second method of VanRaden [70]:

$$
A_{j k}=\frac{1}{M} \sum_{i=1}^{M} \frac{\left(x_{i j}-2 p_{i}\right)\left(x_{i k}-2 p_{i}\right)}{2 p_{i}\left(1-p_{i}\right)}
$$

Where $A_{j k}$ is off-diagonal element for animal $j$ and animal $k$ or represents the diagonal element if $\mathrm{j}=\mathrm{k}$, with genotype codes of $x_{\mathrm{ij}}=0,1,2$ for $A_{1} A_{1}, A_{1} A_{2}$, and $A_{2} A_{2}$, respectively. $p_{j}$ is the allele frequency of $A_{2}$ at locus $j$ calculated based on SNP genotype data of the population and $M$ is the number of SNPs in the panel. The $\mathbf{G}$ matrix was constructed using all DNA variants in the 7.8 M DNA variant panel, i.e. mixed linear model with candidate marker included (MLMi) so that the $\mathbf{G}$ matrix was constructed based on all 30,155 SNPs for the $50 \mathrm{~K}$ SNP GWAS and on all the 7,853,211 DNA variants for the 7.8 M SNP panel GWAS.

For each SNP or DNA variant, the allele substitution effect and its $P$-value were estimated using the GCTA package $[67,68]$. The phenotypic variance explained by a single SNP was calculated by $\operatorname{Var}(\%)=\frac{2 p q \beta^{2}}{S^{2}} * 100 \%$, where $p$ and $q$ denote the minor frequency and major frequency for the SNP, respectively, $\beta$ is the SNP allele substitution effect, and $2 p q \beta^{2}$ is the additive genetic variance, and $S^{2}$ is phenotypic variance. DNA variants (or SNPs) that have a nominal $P$-value $<0.005$ were considered as suggestive QTLs as proposed by Benjamin et al. [26], while SNPs with a nominal $P$-value $<10^{-5}$ were classified as significant QTLs based on the recommendation of the Wellcome Trust Case Control Consortium [71]. SNPs that have a nominal $P$-value $<10^{-5}$ were 
further examined for the genome-wise false discovery rate (FDR), which was calculated following the Benjamini-Hochberg procedure for each SNP [27]. At each significance threshold when multiple SNPs within a window of $70 \mathrm{~kb}$ upstream and downstream are significantly associated with a trait, the SNP with the lowest nominal $P$-value was identified as the lead SNP whereas the remaining SNPs were classified as support SNPs. A $70 \mathrm{~kb}$ window was chosen for this study as this was the chromosomal length within which a high LD phase correlation ( $>0.77$ ) was maintained in a Canadian multibreed population [54].

Heritability of a trait was estimated using GREML-LDMS $[72,73]$ for both the $50 \mathrm{~K}$ SNP panel and the 7.8 M DNA variant panel. In GREML-LDMS, DNA variants were stratified into four groups by their mean LD scores within a sliding window, representing the first, second, third, and fourth quartiles of the mean LD score distribution. A GRM was subsequently constructed with DNA variants in each group. The GRMs were then fitted simultaneously into the above statistical model without the single DNA variant effect and the variance components were estimated via a restricted maximum likelihood (REML) as implemented in the GCTA package $[67,69,74,75]$. The genomic heritability of a trait was calculated as a ratio of the total additive genetic variance over the phenotypic variance of the trait.

\section{Inference of genetic architecture based on GWAS results}

Distribution of SNP effects of each carcass trait was generated by plotting squared allele substitution effects of all DNA variants in the $7.8 \mathrm{M}$ DNA variant panel, and by plotting the amount of additive genetic variances explained by single DNA variants in the panel. The average of squared allele substitution effects was obtained for each of the 9 broad functional classes (Table 3) by summing all squared allele substitution effects within the broad functional class divided by the total number of DNA variants within the functional class. The additive genetic variance accounted for by each of the 9 functional classes was estimated by fitting the GRM constructed based on the DNA variants of the functional class and the GRM constructed based on the DNA variants of all other functional classes simultaneously in the statistical model using the GCTA package. The amount of additive genetic variance explained per sequence variant within a functional class was obtained by the additive genetic variance captured by the functional class divided by the number of DNA variants in the class.

\section{Candidate gene identification and functional enrichment analyses}

Lead SNPs with a FDR $<0.10$ were selected to search for candidate genes. Subsequently, genes located within $70 \mathrm{~kb}$ upstream and downstream of the lead SNP were considered candidate genes associated with the trait based on SNP annotation information from the UMD3.1 bovine genome assembly from the Ensembl genome browser (https://www. ensembl.org/). Ingenuity Pathway Analysis (IPA) (Ingenuity Systems, Redwood City, CA; https://www.qiagenbioinformatics.com/products/ingenuity-pathway-analysis/) (IPA Spring 2019 release) was used for the functional enrichment analyses of the candidate genes identified via the GWAS. Briefly, for the genes with known human orthologues from Ensembl, their gene IDs were replaced with their human orthologous gene IDs, whereas those without human orthologues their bovine gene IDs were maintained in the gene list. These Ensembl gene IDs were then used as input gene identifiers in IPA and a core analysis was performed on the genes that were mapped to the IPA knowledge base database. With the list of candidate genes and genes mapped to the human orthologues, enhanced molecular processes and gene network were inferred using IPA. Molecular, cellular, and biological processes or functions were significantly enriched if the $P$-value for the overlap comparison test between the input gene list and the IPA knowledge base database for a given biological function was less than 0.05 . Additionally, genes and biological processes or sub-functions' interaction networks within the most significant molecular and cellular function were produced to show possible biological networks for the trait.

\section{Supplementary information}

Supplementary information accompanies this paper at https://doi.org/10. 1186/s12864-019-6273-1.

\begin{abstract}
Additional file 1: This file contains Additional figures including Figure S1. Distribution of SNP allele substitution effects (left) and additive genetic variances explained by individual SNP (right) based on GWAS of imputed 7.8 M whole genome sequence (WGS) variants for HCW, AFAT, REA, LMY, and AFAT; Figure S2. Cellular and molecular processes for HCW, AFAT, REA, LMY, and CMAR; Figure S3. Gene network for major gene expression, Carbohydrate/lipid metabolisms and Cell Morphology for HCW, AFAT, REA, LMY, and CMAR; Figure S4. Venn diagram showing the overlapped lead significant SNPs (a) and candidate genes (b) among five carcass merit traits based on the imputed 7.8 M DNA variant GWAS.

Additional file 2: This file contains information of all suggestive significant SNPS/INDELs at $P$-values $<0.005$ based on the $7.8 \mathrm{M}$ WGS variant GWAS for HCW, AFAT, REA, LMY, and CMAR.

Additional file 3: This file contains additional Tables including Table S1. Functional annotation of 7.8 M WGS variants along with the number of variants in each class, classification of SNP functions, percentage of WGS and 9 functional class assignments; Table S2. Functional annotation of $50 \mathrm{~K}$ SNP genotypes after quality control along with the number of variants in each class, classification of SNP functions, percentage of WGS and 9 functional class assignments; Table S3. SNP functional annotation of all DNA variants $(38,318,974)$ based on DNA variants of the 1000 bulls genome project; Table S4. List of lead SNPs that were overlapped with QTLs published in the Cattle QTL database within $1 \mathrm{M}$ bp up or $1 \mathrm{M}$ bp downstream for HCW, AFAT, REA, LMY, and CMAR.
\end{abstract}

Abbreviations

ADG: Average daily gain; AFAT: Average backfat thickness; BTA: Bos taurus autosome; CMAR: Carcass marbling score; DMI: Dry matter intake;

DNA: Deoxyribonucleic acid; FDR: Genome-wide false discovery rate; 
GRM: Genomic relationship matrix; GWAS: Genome-wide association study; HCW: Hot carcass weight; HWT: Hardy-Weinberg equilibrium test; INDEL: Insertion and deletion; LD: Linkage disequilibrium; LMY: Lean meat yield; MAF: Minor allele frequency; QTL: Quantitative trait loci; REA: Rib eye area; SNP: Single nucleotide polymorphism; WGS: Whole genome sequence

\section{Acknowledgements}

The authors thank Dr. John Crowley for help on phenotype data compilation, Dr. Kirill Krivushin for assistance on annotation of whole genome sequence variants to functional classes, and Dr. Zhiliang Hu for help on searching and presentation of cattle QTL information. The data analysis was enabled in part by support provided by WestGrid (http://www.westgrid. ca) and Compute Canada Calcul Canada (http://www.computecanada.ca).

\section{Authors' contributions}

$\mathrm{CL}$ conceived, designed, and oversaw the study. LC, MV, CL consolidated original genotype and phenotypic data. GP, JB, PS participated in the design of the study and contributed to acquisition of data. LC, MV performed imputation from $50 \mathrm{~K}$ to AffyHD. YW conducted imputation from AffyHD to WGS variants. PS annotated WGS variants to functional classes. YW and FZ performed data analyses including GWAS. RM performed functional enrichment analyses. CL and YW interpreted the data and results, and wrote the paper. All authors read, commented and approved the final manuscript

\section{Funding}

This work was supported by the Alberta Livestock and Meat Agency (ALMA)/ Alberta Agriculture and Forestry (AAF) (2014F047R) and in part by the Genome Alberta and AAF project (GAB-A3GP37). The ALMA/AAF project provided financial support to personnel to conduct genotypic and phenotypic data consolidation, SNP imputation, SNP functional annotation, GWAS, enriched biological function analyses, and manuscript writing. The Genome Alberta/AAF project supported in part on the enriched biological function analyses and manuscript writing. The original SNP genotype and phenotypic data were collected under multiple research projects led by Dr. Changxi Li (Angus and Charolais), Drs. Stephen Moore and Graham Plastow (Kinsella Composite), Dr. Stephen Miller (Elora) under the Bovine Genome Project, and Dr. John Basarab (PG1 and TXX) under the Phenomic Gap Project. These multiple research projects were funded by Alberta Funding Consortium, Alberta Livestock and Meat Agency, Alberta Innovates Biosolutions, Western Economic Development, Genome Canada, and Genome Alberta, and supported through in-kind and cash contributions from Agriculture and Agri-Food Canada (AAFC), Alberta Agriculture and Forestry, University of Alberta, University of Guelph, Beef Cattle Research Council, Beefbooster Inc., Deseret Ranches of Alberta, Three Cross Ranches, Namaka Farms, Growsafe Systems Inc., Canadian Angus Association, Canadian Charolais Association, and Canadian Hereford Association. The full genome sequence information from the Canadian Cattle Genome Project was obtained with support from Genome Canada, Genome Alberta, Alberta Livestock and Meat Agency Ltd., Western Economic Diversification, and Alberta Innovates Bio Solutions, with cattle samples contributed by industry partners including the Canadian Angus, Simmental, Hereford, Charolais, Limousin, Gelbvieh associations, Beefbooster Inc., and with collaborative assistance provided by AgResearch, BCRC, BIO, Semex, SRUC, Teagasc, and the USDA-ARS. The China Scholarship Council provided financial support to FZ to conduct the study under the AAFC-MOE joint PhD research program. The funding bodies played no role in the design of the study and collection, analysis, and interpretation of data and in writing the manuscript.

\section{Availability of data and materials}

The datasets supporting the results of this article are included within the article and its additional files. The original genotype and phenotype data sets are available for non-commercial purposes following the execution of a materials transfer agreement. Whole genome sequence data collected for the cattle populations in this study and used in imputation is available from the NCBI SRA database under BioProjects PRJNA176557 and PRJNA256210.

\section{Ethics approval and consent to participate}

Data on the animals used in this study were collected in previous projects and all the animals were cared for according to the guidelines established by the Canadian Council on Animal Care (1993) and approved by local animal user committees including University of Alberta Faculty Animal Policy and Welfare Committee (2002-46B for the Bovine Genome Project and AUP00000777 for the Kinsella Breeding Project) and the AAFC Lacombe Research Center Animal Care Committee (200703 and 200807 for the Phenomic Gap Project). The reference sequence data for imputation were obtained from the 1000 bull genomes project under the Canadian Cattle Genome Project. The study was approved by the Agriculture and Agri-Food Canada Lacombe Research Proposal Review committee (J-000960).

\section{Consent for publication}

Not applicable.

\section{Competing interests}

PS serves as an Associate Editor for BMC Genomics. Beyond this association, the authors declare that they have no competing interests.

\section{Author details}

${ }^{1}$ Lacombe Research and Development Centre, Agriculture and Agri-Food Canada, Lacombe, AB, Canada. ${ }^{2}$ Department of Agricultural, Food and Nutritional Science, University of Alberta, Edmonton, AB, Canada. ${ }^{3}$ State Key Laboratory for Swine Genetics, Breeding and Production Technology, Jiangxi Agricultural University, Nanchang, Jiangxi, China. ${ }^{4}$ Present Address: Institute of Translational Medicine, Nanchang University, Nanchang, Jiangxi, China. ${ }^{5}$ Alberta Agriculture and Forestry, Lacombe Research and Development Centre, 6000 C\&E Trail, Lacombe, AB, Canada.

\section{Received: 26 June 2019 Accepted: 12 November 2019}

\section{Published online: 13 January 2020}

\section{References}

1. Schröder UJ, Staufenbiel R. Invited review: methods to determine body fat reserves in the dairy cow with special regard to ultrasonographic measurement of backfat thickness. J Dairy Sci. 2006;89(1):1-14.

2. Matukumalli LK, Lawley CT, Schnabel RD, Taylor JF, Allan MF, Heaton MP, O'Connell J, Moore SS, Smith TP, Sonstegard TS, et al. Development and characterization of a high density SNP genotyping assay for cattle. PLoS One. 2009;4(4):e5350.

3. Meuwissen THE, Hayes BJ, Goddard ME. Prediction of total genetic value using genome-wide dense marker maps. Genetics. 2001;157(4):1819-29.

4. Dekkers JCM, Hospital F. The use of molecular genetics in the improvement of agricultural populations. Nat Rev Genet. 2002;3(1):22-32.

5. Chen L, Vinsky M, Li C. Accuracy of predicting genomic breeding values for carcass merit traits in Angus and Charolais beef cattle. Anim Genet. 2015; 46(1):55-9.

6. Mehrban H, Lee DH, Moradi MH, IICho C, Naserkheil M, Ibanez-Escriche N. Predictive performance of genomic selection methods for carcass traits in Hanwoo beef cattle: impacts of the genetic architecture. Genet Sel Evol. 2017:49(1):1.

7. Ogawa S, Matsuda H, Taniguchi Y, Watanabe T, Kitamura Y, Tabuchi I, Sugimoto Y, Iwaisaki H. Genomic prediction for carcass traits in Japanese black cattle using single nucleotide polymorphism markers of different densities. Anim Prod Sci. 2017;57(8):1631-6.

8. Hayes BJ, Bowman PJ, Chamberlain AC, Verbyla K, Goddard ME. Accuracy of genomic breeding values in multi-breed dairy cattle populations. Genet Sel Evol. 2009:41(1):51.

9. Snelling WM, Cushman RA, Fortes MRS, Reverter A, Bennett GL, Keele JW, Kuehn LA, McDaneld TG, Thallman RM, Thomas MG. PHYSIOLOGY AND ENDOCRINOLOGY SYMPOSIUM: how single nucleotide polymorphism chips will advance our knowledge of factors controlling puberty and aid in selecting replacement beef females. J Anim Sci. 2012;90(4):1152-65.

10. Stone RT, Keele JW, Shackelford SD, Kappes SM, Koohmaraie M. A primary screen of the bovine genome for quantitative trait loci affecting carcass and growth traits. J Anim Sci. 1999;77(6):1379-84.

11. Casas E, Shackelford SD, Keele JW, Koohmaraie M, Smith TPL, Stone RT, Detection of quantitative trait loci for growth and carcass composition in cattle. J Anim Sci. 2003;81(12):2976-83.

12. Goddard ME, Hayes BJ. Mapping genes for complex traits in domestic animals and their use in breeding programmes. Nat Rev Genet. 2009;10(6): 381-91.

13. Boichard D, Chung H, Dassonneville R, David X, Eggen A, Fritz S, Gietzen KJ, Hayes BJ, Lawley CT, Sonstegard TS, et al. Design of a bovine low-density SNP array optimized for imputation. PLoS One. 2012;7(3):e34130. 
14. $\mathrm{Hu} Z \mathrm{~L}$, Park CA, Reecy JM. Building a livestock genetic and genomic information knowledgebase through integrative developments of animal QTLdb and CorrDB. Nucleic Acids Res. 2019;47(D1):D701-10.

15. Beever JE, George PD, Fernando RL, Stormont CJ, Lewin HA. Associations between genetic markers and growth and carcass traits in a paternal halfsib family of Angus cattle. J Anim Sci. 1990;68(2):337.

16. Grobet L, Royo Martin LJ, Poncelet D, Pirottin D, Brouwers B, Riquet J, Schoeberlein A, Dunner S, Ménissier F, Massabanda J, et al. A deletion in the bovine myostatin gene causes the double-muscled phenotype in cattle. Nat Genet. 1997:17(1):71-4.

17. Keele JW, Shackelford SD, Kappes SM, Koohmaraie M, Stone RT. A region on bovine chromosome 15 influences beef longissimus tenderness in steers. J Anim Sci. 1999;77(6):1364.

18. Casas E, Stone RT, Keele JW, Shackelford SD, Kappes SM, Koohmaraie M. A comprehensive search for quantitative trait loci affecting growth and carcass composition of cattle segregating alternative forms of the myostatin gene. J Anim Sci. 2001;79(4):854-60.

19. Moore SS, Li C, Basarab J, Snelling WM, Kneeland J, Murdoch B, Hansen C, Benkel B. Fine mapping of quantitative trait loci and assessment of positional candidate genes for backfat on bovine chromosome 14 in a commercial line of Bos taurus. J Anim Sci. 2003:81(8):1919-25.

20. Li C, Basarab J, Snelling WM, Benkel B, Kneeland J, Murdoch B, Hansen C, Moore SS. Identification and fine mapping of quantitative trait loci for backfat on bovine chromosomes 2, 5, 6, 19, 21, and 23 in a commercial line of Bos taurus. J Anim Sci. 2004;82(4):967-72.

21. Nkrumah JD, Li C, Basarab JB, Guercio S, Meng Y, Murdoch B, Hansen C, Moore SS. Association of a single nucleotide polymorphism in the bovine leptin gene with feed intake, feed efficiency, growth, feeding behaviour, carcass quality and body composition. Can J Anim Sci. 2004;84(2):211-9.

22. Daetwyler HD, Capitan A, Pausch H, Stothard P, van Binsbergen R, Brøndum RF, Liao X, Djari A, Rodriguez SC, Grohs C, et al. Whole-genome sequencing of 234 bulls facilitates mapping of monogenic and complex traits in cattle. Nat Genet. 2014;46(8):858-65.

23. Zhang F, Wang Y, Mukiibi R, Chen L, Vinsky M, Plastow G, Basarab J, Stothard P, Li C. Genetic architecture of quantitative traits in beef cattle revealed by genome wide association study of imputed whole genome sequence variants: I: feed efficiency and component traits. BMC Genomics. 2020 https://doi.org/10.1186/s12864-019-6362-1.

24. Ekine-Dzivenu C, Vinsky M, Basarab JA, Aalhus JL, Dugan MER, Li C. Phenotypic and genetic correlations of fatty acid composition in subcutaneous adipose tissue with carcass merit and meat tenderness traits in Canadian beef cattle. J Anim Sci. 2017;95(12):5184-96.

25. Akanno EC, Ekine-Dzivenu C, Chen L, Vinsky M, Abo-Ismail MK, MacNeil MD, Plastow G, Basarab J, Li C, Fitzsimmons C. Evaluation of a genomicenhanced sorting system for feeder cattle. J Anim Sci. 2019;97(3):1066-75.

26. Benjamin DJ, Berger JO, Johannesson M, Nosek BA, Wagenmakers EJJ, Berk R, Bollen KA, Brembs B, Brown L, Camerer C, et al. Redefine statistical significance. Nat Hum Behav. 2018;2(1):6-10

27. Benjamini $Y$, Hochberg Y. Controlling the false discovery rate: a practical and powerful approach to multiple testing. J R Stat Soc Ser B Methodol. 1995;57(1):289-300.

28. Sargolzaei M, Chesnais JP, Schenkel FS. A new approach for efficient genotype imputation using information from relatives. BMC Genomics. 2014;15:478.

29. Bernardes PA, Al-Mamun HA, Suarez M, Lim D, Park B, Gondro C. Imputation accuracy of whole-genome sequence data in Hanwoo cattle. In: Proceedings of the World Congress on Genetics Applied to Livestock Production, Electronic. 2018;11.735.

30. Brøndum R, Guldbrandtsen B, Sahana G, Lund M, Su G. Strategies for imputation to whole genome sequence using a single or multi-breed reference population in cattle. BMC Genomics. 2014;15(1):728.

31. van Binsbergen R, Bink MCAM, Calus MPL, van Eeuwijk FA, Hayes BJ, Hulsegge I, Veerkamp RF. Accuracy of imputation to whole-genome sequence data in Holstein Friesian cattle. Genet Sel Evol. 2014;46(1):41.

32. Chen L, Ekine-Dzivenu C, Vinsky M, Basarab J, Aalhus J, Dugan ME, Fitzsimmons C, Stothard P, Li C. Genome-wide association and genomic prediction of breeding values for fatty acid composition in subcutaneous adipose and longissimus lumborum muscle of beef cattle. BMC Genet. 2015;16:135.

33. Chen L, Schenkel F, Vinsky M, Crews DH Jr, Li C. Accuracy of predicting genomic breeding values for residual feed intake in Angus and Charolais beef cattle. J Anim Sci. 2013;91(10):4669-78.
34. Habier D, Fernando RL, Kizilkaya K, Garrick DJ. Extension of the bayesian alphabet for genomic selection. BMC Bioinformatics. 2011;12(1):186.

35. Lai EC. Micro RNAs are complementary to 3 ' UTR sequence motifs that mediate negative post-transcriptional regulation. Nat Genet. 2002;30(4):363-4.

36. Gu S, Jin L, Zhang FJ, Sarnow P, Kay MA. Biological basis for restriction of microRNA targets to the $3^{\prime}$ untranslated region in mammalian mRNAs. Nat Struct Mol Biol. 2009;16(2):144-50.

37. Rojano E, Seoane P, Ranea JAG, Perkins JR. Regulatory variants: from detection to predicting impact. Brief Bioinform. 2018. p. 1-16.

38. Koufariotis L, Chen Y-P, Bolormaa S, Hayes BJ. Regulatory and coding genome regions are enriched for trait associated variants in dairy and beef cattle. BMC Genomics. 2014;15(1):436.

39. Koufariotis LT, Chen Y-PP, Stothard P, Hayes BJ. Variance explained by whole genome sequence variants in coding and regulatory genome annotations for six dairy traits. BMC Genomics. 2018;19(1):237.

40. Saatchi M, Schnabel RD, Taylor JF, Garrick DJ. Large-effect pleiotropic or closely linked QTL segregate within and across ten US cattle breeds. BMC Genomics. 2014;15(1):442.

41. Lindholm-Perry AK, Sexten AK, Kuehn LA, Smith TPL, King DA, Shackelford SD, Wheeler TL, Ferrell CL, Jenkins TG, Snelling WM, et al. Association, effects and validation of polymorphisms within the NCAPG - LCORL locus located on BTA6 with feed intake, gain, meat and carcass traits in beef cattle. BMC Genet. 2011;12(1):103.

42. Eberlein A, Takasuga A, Setoguchi K, Pfuhl R, Flisikowski K, Fries R, Klopp N, Fürbass R, Weikard R, Kühn C. Dissection of genetic factors modulating fetal growth in cattle indicates a substantial role of the non-SMC condensin I complex, subunit G (NCAPG) gene. Genetics. 2009;183(3):951-64.

43. Setoguchi K, Watanabe T, Weikard R, Albrecht E, Kühn C, Kinoshita A, Sugimoto Y, Takasuga A. The SNP C.1326T>G in the non-SMC condensin I complex, subunit G (NCAPG) gene encoding a p.lle442Met variant is associated with an increase in body frame size at puberty in cattle. Anim Genet. 2011:42(6):650-5.

44. Setoguchi K, Furuta M, Hirano T, Nagao T, Watanabe T, Sugimoto $Y$, Takasuga A. Cross-breed comparisons identified a critical 591-kb region for bovine carcass weight QTL (CW-2) on chromosome 6 and the lle-442-met substitution in NCAPG as a positional candidate. BMC Genet. 2009;10(1):43.

45. Sahana G, Höglund JK, Guldbrandtsen B, Lund MS. Loci associated with adult stature also affect calf birth survival in cattle. BMC Genet. 2015;16(1):47.

46. Patwari P, Emilsson V, Schadt Eric E, Chutkow William A, Lee S, Marsili A, Zhang Y, Dobrin R, Cohen David E, Larsen PR, et al. The arrestin domaincontaining 3 protein regulates body mass and energy expenditure. Cell Metab. 2011;14(5):671-83.

47. Patwari $P$, Lee RT. An expanded family of arrestins regulate metabolism. Trends Endocrinol Metab. 2012;23(5):216-22

48. Karim L, Takeda H, Lin L, Druet T, Arias JAC, Baurain D, Cambisano N, Davis $S R$, Farnir F, Grisart B, et al. Variants modulating the expression of a chromosome domain encompassing PLAG1 influence bovine stature. Nat Genet. 2011;43(5):405-13.

49. Fink T, Tiplady K, Lopdell T, Johnson T, Snell RG, Spelman RJ, Davis SR, Littlejohn MD. Functional confirmation of PLAG1 as the candidate causative gene underlying major pleiotropic effects on body weight and milk characteristics. Sci Rep. 2017;7:44793.

50. Bouwman AC, Daetwyler HD, Chamberlain AJ, Ponce $\mathrm{CH}$, Sargolzaei M, Schenkel FS, Sahana G, Govignon-Gion A, Boitard S, Dolezal M, et al. Meta-analysis of genome-wide association studies for cattle stature identifies common genes that regulate body size in mammals. Nat Genet. 2018;50(3):362-7.

51. Wang X, Zhang Y, Zhang X, Wang D, Jin G, Li B, Xu F, Cheng J, Zhang F, Wu $S$, et al. The comprehensive liver transcriptome of two cattle breeds with different intramuscular fat content. Biochem Biophys Res Commun. 2017:490(3):1018-25.

52. Mukiibi R, Vinsky M, Keogh K, Stothard P, Waters S, Li C. Transcriptome analysis of liver tissues in Charolais steers with divergent marbling phenotypes. In: Proceedings of the World Congress on Genetics Applied to Livestock Production, Electronic, vol. 11; 2018. p. 626.

53. Nkrumah JD, Keisler DH, Crews DH, Basarab JA, Wang Z, Li C, Price MA, Okine EK, Moore SS. Genetic and phenotypic relationships of serum leptin concentration with performance, efficiency of gain, and carcass merit of feedlot cattle1. J Anim Sci. 2007:85(9):2147-55.

54. Lu D, Sargolzaei M, Kelly M, Li C, Vander Voort G, Wang Z, Plastow G, Moore S, Miller SP. Linkage disequilibrium in Angus, Charolais, and crossbred beef cattle. Front Genet. 2012;3:152. 
55. Mao F, Chen L, Vinsky M, Okine E, Wang Z, Basarab J, Crews DH, Li C. Phenotypic and genetic relationships of feed efficiency with growth performance, ultrasound, and carcass merit traits in Angus and Charolais steers. J Anim Sci. 2013;91(5):2067-76.

56. Zhang F, Ekine-Dzivenu C, Vinsky M, Basarab J, Aalhus J, Dugan M, Li C. Phenotypic and genetic relationships of residual feed intake measures and their component traits with fatty acid composition in subcutaneous adipose of beef cattle. J Anim Sci. 2017;95(7):2813-24.

57. Basarab JA, Price MA, Aalhus JL, Okine EK, Snelling WM, Lyle KL. Residual feed intake and body composition in young growing cattle. Can J Anim Sci. 2003;83(2):189-204.

58. Nkrumah JD, Crews DH, Basarab JA, Price MA, Okine EK, Wang Z, Li C, Moore SS. Genetic and phenotypic relationships of feeding behavior and temperament with performance, feed efficiency, ultrasound, and carcass merit of beef cattle. J Anim Sci. 2007;85(10):2382-90

59. Basarab JAA, Colazo MGG, Ambrose DJJ, Novak S, McCartney D, Baron VSS. Residual feed intake adjusted for backfat thickness and feeding frequency is independent of fertility in beef heifers. Can J Anim Sci. 2011;91(4):573-84.

60. Lu D, Sargolzaei M, Kelly M, Vander Voort G, Wang Z, Mandell I, Moore S, Plastow G, Miller SP. Genome-wide association analyses for carcass quality in crossbred beef cattle. BMC Genet. 2013;14:80.

61. Chen L, Mao F, Crews DH Jr, Vinsky M, Li C. Phenotypic and genetic relationships of feeding behavior with feed intake, growth performance, feed efficiency, and carcass merit traits in Angus and Charolais steers. J Anim Sci. 2014;92(3):974-83.

62. Akanno EC, Plastow G, Woodward BW, Bauck S, Okut H, Wu XLXL, Sun C, Aalhus JL, Moore SS, Miller SP, et al. Reliability of molecular breeding values for Warner-Bratzler shear force and carcass traits of beef cattle - an independent validation study. J Anim Sci. 2014;92(7):2896-904.

63. Browning SR, Browning BL. Rapid and accurate haplotype phasing and missing-data inference for whole-genome association studies by use of localized haplotype clustering. Am J Hum Genet. 2007;81 (5):1084-97.

64. Stothard P, Liao X, Arantes AS, De Pauw M, Coros C, Plastow GS, Sargolzaei M, Crowley JJ, Basarab JA, Schenkel F, et al. A large and diverse collection of bovine genome sequences from the Canadian cattle genome project. Gigascience. 2015;4:49.

65. Grant JR, Arantes AS, Liao X, Stothard P. In-depth annotation of SNPs arising from resequencing projects using NGS-SNP. Bioinformatics. 2011;27(16): 2300-1.

66. Alexander DH, Novembre J, Lange K. Fast model-based estimation of ancestry in unrelated individuals. Genome Res. 2009;19(9):1655-64

67. Yang J, Lee SH, Goddard ME, Visscher PM. GCTA: a tool for genome-wide complex trait analysis. Am J Hum Genet. 2011;88(1):76-82.

68. Yang J, Zaitlen NA, Goddard ME, Visscher PM, Price AL. Advantages and pitfalls in the application of mixed-model association methods. Nat Genet. 2014;46(2):100

69. Yang J, Benyamin B, McEvoy BP, Gordon S, Henders AK, Nyholt DR, Madden PA, Heath AC, Martin NG, Montgomery GW, et al. Common SNPs explain a large proportion of the heritability for human height. Nat Genet. 2010;42(7):565.

70. VanRaden PM. Efficient methods to compute genomic predictions. J Dairy Sci. 2008;91(11):4414-23.

71. Consortium TWTCC, Burton PR, Clayton DG, Cardon LR, Craddock N, Deloukas P, Duncanson A, Kwiatkowski DP, McCarthy MI, Ouwehand WH, et al. Genome-wide association study of 14,000 cases of seven common diseases and 3,000 shared controls. Nature. 2007;447:661.

72. Yang J, Bakshi A, Zhu Z, Hemani G, Vinkhuyzen AAE, Lee SH, Robinson MR, Perry JRB, Nolte IM, van Vliet-Ostaptchouk JV, et al. Genetic variance estimation with imputed variants finds negligible missing heritability for human height and body mass index. Nat Genet. 2015;47(10):1114.

73. Yang J, Zeng J, Goddard ME, Wray NR, Visscher PM. Concepts, estimation and interpretation of SNP-based heritability. Nat Genet. 2017:49(9):1304-10.

74. Yang J, Manolio TA, Pasquale LR, Boerwinkle E, Caporaso N, Cunningham JM, Md A, Feenstra B, Feingold E, Hayes MG, et al. Genome partitioning of genetic variation for complex traits using common SNPs. Nat Genet. 2011;43(6):519.

75. Lee SH, Wray NR, Goddard ME, Visscher PM. Estimating missing heritability for disease from genome-wide association studies. Am J Hum Genet. 2011; 88(3):294-305

\section{Publisher's Note}

Springer Nature remains neutral with regard to jurisdictional claims in published maps and institutional affiliations.

\section{Ready to submit your research? Choose BMC and benefit from}

- fast, convenient online submission

- thorough peer review by experienced researchers in your field

- rapid publication on acceptance

- support for research data, including large and complex data types

- gold Open Access which fosters wider collaboration and increased citations

- maximum visibility for your research: over $100 \mathrm{M}$ website views per year

At BMC, research is always in progress.

Learn more biomedcentral.com/submissions 